\title{
Penalization of a Positively Recurrent Diffusion by an Exponential Function of its Local Time
}

\author{
by
}

\author{
Christophe Profeta
}

\begin{abstract}
Using Krein's theory of strings, we penalize here a large class of positively recurrent diffusions by an exponential function of their local time. After a brief study of the processes so penalized, we show that on this example the principle of penalization can be iterated, and that the family of probabilities we get forms a group. We conclude by an application to Bessel processes of dimension $\delta \in] 0,2[$ which are reflected at 1 .
\end{abstract}

2010 Mathematics Subject Classification: Primary 60J60; Secondary 60J55, 60J65.

Keywords: penalization, positively recurrent diffusions, local time, Krein's theory, Bessel processes.

\section{$\S 1$. Introduction}

1. Let $b \in] 0,+\infty]$. We consider a linear regular diffusion $X$ taking values in $I=[0, b)$, on natural scale, and with 0 an instantaneously reflecting boundary. Let $\mathbb{P}_{x}$ and $\mathbb{E}_{x}$ denote, respectively, the probability measure and the expectation associated with $X$ when started from $x \geq 0$. We assume that $X$ is defined on the canonical space $\Omega:=\mathcal{C}\left(\mathbb{R}_{+} \rightarrow \mathbb{R}_{+}\right.$) (where $\mathbb{R}_{+}:=[0,+\infty[$ ), and we denote by $\left(\mathcal{F}_{t}, t \geq 0\right)$ its natural filtration, with $\mathcal{F}_{\infty}:=\bigvee_{t \geq 0} \mathcal{F}_{t}$

Let us start by giving a definition of penalization (see also Theorem 3.1):

Definition 1.1. Let $\left(\Gamma_{t}, t \geq 0\right)$ be a measurable process taking positive values and such that $0<\mathbb{E}_{x}\left[\Gamma_{t}\right]<\infty$ for every $t>0$ and every $x \in I$. We say that the process $\left(\Gamma_{t}, t \geq 0\right)$ satisfies the penalization principle if there exists a probability measure $\mathbb{Q}_{x}$ defined on $\left(\Omega, \mathcal{F}_{\infty}\right)$ such that

$$
\forall s>0, \forall \Lambda_{s} \in \mathcal{F}_{s}, \quad \lim _{t \rightarrow+\infty} \frac{\mathbb{E}_{x}\left[1_{\Lambda_{s}} \Gamma_{t}\right]}{\mathbb{E}_{x}\left[\Gamma_{t}\right]}=\mathbb{Q}_{x}\left(\Lambda_{s}\right) .
$$

Communicated by Y. Takahashi. Received May 14, 2009. Revised February 16, 2010.

C. Profeta: IECN, Nancy-Université, CNRS, Boulevard des Aiguillettes, B.P. 70239 , F-54506 Vandœuvre-lès-Nancy, France;

e-mail: profeta@iecn.u-nancy.fr

(c) 2010 Research Institute for Mathematical Sciences, Kyoto University. All rights reserved. 
This problem has been thoroughly studied by B. Roynette, P. Vallois and M. Yor when $\mathbb{P}_{x}$ is the Wiener measure (see RVY06 for a synthesis and further references). Let $\left(L_{t}, t \geq 0\right)$ be the local time of $X$ at 0 , and $\left(\tau_{l}, l \geq 0\right)$ the rightcontinuous inverse of $L$ :

$$
\tau_{l}:=\inf \left\{t \geq 0 ; L_{t}>l\right\}
$$

Recently, P. Salminen and P. Vallois [SV09] have proved that the penalization principle holds when $\left(\Gamma_{t}=h\left(L_{t}\right), t \geq 0\right)$ with $h$ a non-negative and non-increasing function, under the assumption that the Lévy measure of the subordinator $\left(\tau_{l}, l \geq 0\right)$ is subexponential (see Remark 5). Here, we are interested in extending these results to other diffusions, with weight process $\left(\Gamma_{t}:=e^{\alpha L_{t}}, t \geq 0\right)$ for $\alpha \in \mathbb{R}$. We will focus mainly on the positively recurrent case (in Sections 2 to 5 , which has not been studied yet. Other cases will be briefly dealt with in Section 6, where we will see how, in the null recurrent case, the assumption of subexponentiality appears naturally.

2. Our approach of penalization with $\left(\Gamma_{t}:=e^{\alpha L_{t}}, t \geq 0\right)$ is based on the rate of decay (or growth) of $\mathbb{E}_{x}\left[e^{\alpha L_{t}}\right]$ as $t$ tends to infinity. But before stating our main results, we need a few notations. Let $m$ denote the speed measure of $X$. We assume that $m$ is strictly positive in the vicinity of 0 and does not have atoms (see A. N. Borodin and P. Salminen BS02, Chapter II] for the definition of the main attributes of a linear diffusion). It is known that $X$ admits a transition density $p(t, x, y)$ (with respect to $m$ ) that is jointly continuous and symmetric in $x$ and $y$ (see IM74, Chapter 4, p. 149]). We also introduce its resolvent kernel:

$$
R_{\lambda}(x, y)=\int_{0}^{\infty} e^{-\lambda t} p(t, x, y) d t
$$

Now, let $\alpha>0$. We assume that $X$ is a recurrent diffusion reflected on $[0, b]$ and such that $b+m([0, b[)<\infty$. This hypothesis implies in particular that $X$ is positively recurrent. In this case, the equation

$$
\alpha+\frac{1}{R_{-r^{2}}(0,0)}=0
$$

admits a countable number of solutions, they are all real, and we denote by $r^{2}$ the one of smallest modulus (see Lemma 2.3. Similarly, we denote by $\rho^{2}$ the unique solution in $\mathbb{R}_{+}$of the equation

$$
-\alpha+\frac{1}{R_{\rho^{2}}(0,0)}=0 .
$$

We can now give our first theorem: 
Theorem 1.1. Let $\alpha>0$ and let $r^{2}, \rho^{2}$ be defined by equations 1.2 and 1.3 . Then:

(i) Under Assumption 2.1 we have (see Section 2)

$$
\mathbb{E}_{x}\left[e^{-\alpha L_{t}}\right] \underset{t \rightarrow+\infty}{\sim} \frac{1}{r^{2}} R_{-r^{2}}(0, x) \frac{1}{\left.\frac{\partial}{\partial z} R_{z}(0,0)\right|_{z=-r^{2}}} \exp \left(-r^{2} t\right) .
$$

(ii) Under Assumption 3.1 we have (see Subsection 3.5

$$
\mathbb{E}_{x}\left[e^{\alpha L_{t}}\right] \underset{t \rightarrow+\infty}{\sim}-\frac{1}{\rho^{2}} R_{\rho^{2}}(0, x) \frac{1}{\left.\frac{\partial}{\partial z} R_{z}(0,0)\right|_{z=\rho^{2}}} \exp \left(\rho^{2} t\right) .
$$

This result will enable us to obtain our penalization principle, under the assumption that $m(d x)=m(x) d x$ :

Theorem 1.2. Let $\alpha>0$ and let $r^{2}, \rho^{2}$ be defined by equations 1.2 and 1.3 . For $x \in[0, b]$, the processes

$$
\left(M_{t}^{(-\alpha)}:=\exp \left(r^{2} t-\alpha L_{t}\right) \frac{R_{-r^{2}}\left(0, X_{t}\right)}{R_{-r^{2}}(0, x)}, t \geq 0\right)
$$

and

$$
\left(M_{t}^{(+\alpha)}:=\exp \left(-\rho^{2} t+\alpha L_{t}\right) \frac{R_{\rho^{2}}\left(0, X_{t}\right)}{R_{\rho^{2}}(0, x)}, t \geq 0\right)
$$

are continuous, strictly positive $\mathbb{P}_{x}$-martingales which converge to 0 as $t \rightarrow+\infty$. Moreover, under Assumptions 2.1 and 3.1, the penalization principle holds:

(i) Let $s>0$ and $x \in[0, b]$. For all $\Lambda_{s} \in \mathcal{F}_{s}$, we have

$$
\lim _{t \rightarrow+\infty} \frac{\mathbb{E}_{x}\left[1_{\Lambda_{s}} e^{ \pm \alpha L_{t}}\right]}{\mathbb{E}_{x}\left[e^{ \pm \alpha L_{t}}\right]}=\mathbb{E}_{x}\left[1_{\Lambda_{s}} M_{s}^{( \pm \alpha)}\right]
$$

(ii) There exists a family $\left(\mathbb{P}_{x}^{( \pm \alpha)}\right)_{x \in[0, b]}$ of probabilities defined on $\left(\Omega, \mathcal{F}_{\infty}\right)$ such that

$$
\mathbb{P}_{x}^{( \pm \alpha)}\left(\Lambda_{u}\right)=\mathbb{E}_{x}\left[1_{\Lambda_{u}} M_{u}^{( \pm \alpha)}\right] \quad \text { for all } u \geq 0 \text { and all } \Lambda_{u} \in \mathcal{F}_{u}
$$

We now study the law of the coordinate process under $\mathbb{P}^{( \pm \alpha)}$ :

Theorem 1.3. Let $\alpha>0$, let $r^{2}, \rho^{2}$ be defined by equations (1.2) and (1.3), and suppose that Assumptions 2.1 and 3.1 hold. Then:

(i) Under $\mathbb{P}^{( \pm \alpha)}$, the coordinate process $X$ is a diffusion with infinitesimal generator respectively given by

$$
\left\{\begin{array}{l}
\mathcal{G}^{(-\alpha)} f(x):=\frac{1}{m(x)} f^{\prime \prime}(x)+\frac{2}{m(x) R_{-r^{2}}(0, x)} \frac{\partial R_{-r^{2}}(0, x)}{\partial x} f^{\prime}(x), \\
\mathcal{G}^{(+\alpha)} f(x):=\frac{1}{m(x)} f^{\prime \prime}(x)+\frac{2}{m(x) R_{\rho^{2}}(0, x)} \frac{\partial R_{\rho^{2}}(0, x)}{\partial x} f^{\prime}(x),
\end{array}\right.
$$


defined on the domain

$$
\mathcal{D}\left(\mathcal{G}^{( \pm \alpha)}\right):=\left\{f ; \mathcal{G}^{( \pm \alpha)} f \in \mathcal{C}_{\mathrm{b}}([0, b]), f^{\prime}\left(0^{+}\right)=f^{\prime}\left(b^{-}\right)=0\right\} .
$$

(ii) Under $\mathbb{P}^{( \pm \alpha)}$, the density of the Lévy measure of the subordinator $\tau$ is given by

$$
\left\{\begin{array}{l}
n^{(-\alpha)}(u)=e^{r^{2} u} n(u), \\
n^{(+\alpha)}(u)=e^{-\rho^{2} u} n(u),
\end{array}\right.
$$

where $n$ is the density of the Lévy measure of $\tau$ under $\mathbb{P}$.

(iii) $L_{\infty}=\infty \mathbb{P}^{( \pm \alpha)}-$ a.s.

We must stress the fact that (iii) is quite surprising. Indeed, in SV09, the authors prove that for (a large class of) null recurrent diffusions, the penalization principle holds with $\left(e^{-\alpha L_{t}}, t \geq 0\right)(\alpha>0)$, and that the process so penalized is transient (as expected). As shown by Theorem 1.3 this is no longer the case for a positively recurrent diffusion (see also Subsection 3.4).

Some other quantities, such as the speed measure or the scale function of the penalized diffusion, will also be computed during the proof (see Section 3). Note that the expressions in both cases are very similar, and can be deduced formally from each other by replacing $\alpha$ by $-\alpha$ (resp. $-\alpha$ by $\alpha$ ) and $\rho$ by $i r$ (resp. $r$ by $i \rho$ ). A natural idea then is to consider a double penalization: first, we penalize $\mathbb{P}$ with $\left(e^{\alpha L_{t}}, t \geq 0\right)$; second, we penalize $\mathbb{P}^{(\alpha)}$ with $\left(e^{\beta L_{t}}, t \geq 0\right)$. The result is very simple, and can be summarized by a commutative diagram, as in the following theorem:

Theorem 1.4. Let $\alpha, \beta \in \mathbb{R}$. Suppose that Assumptions 2.1 and 3.1 hold. Then the following penalization diagram is commutative:

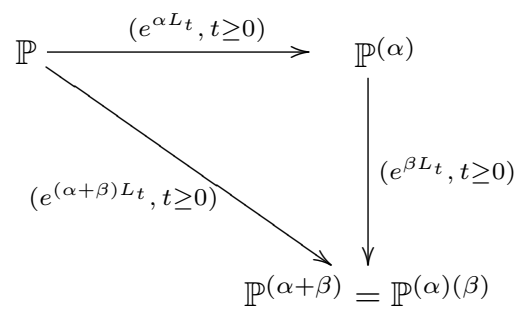

In particular, $\mathbb{P}^{(\alpha)(-\alpha)}=\mathbb{P}$.

Note that this theorem bears a strong resemblance to Proposition 3.2 of PY81 about conditioned diffusions.

Remark 1. If $\left(X_{t}, t \geq 0\right)$ is a linear diffusion whose scale function $s$ is a strictly increasing $\mathcal{C}^{1}$ function such that $s(0)=0$, we have, from the occupation time 
formula, $L_{t}^{0}(X)=L_{t}^{0}(s(X))$. Then

$$
\mathbb{E}_{x}\left[e^{-\alpha L_{t}^{0}(X)}\right]=\mathbb{E}_{s(x)}\left[e^{-\alpha L_{t}^{0}(s(X))}\right] \underset{t \rightarrow+\infty}{\sim} \frac{1}{r^{2}} R_{-r^{2}}(0, s(x)) \frac{1}{\left.\frac{\partial}{\partial z} R_{z}(0,0)\right|_{z=-r^{2}}} e^{-r^{2} t}
$$

and, for $\Lambda_{u} \in \mathcal{F}_{u}$

$$
\begin{aligned}
\mathbb{P}_{x}^{(\alpha, X)}\left(\Lambda_{u}\right) & =\lim _{t \rightarrow+\infty} \frac{\mathbb{E}_{x}\left[1_{\Lambda_{u}} e^{-\alpha L_{t}^{0}(X)}\right]}{\mathbb{E}_{x}\left[e^{-\alpha L_{t}^{0}(X)}\right]}=\lim _{t \rightarrow+\infty} \frac{\mathbb{E}_{s(x)}\left[1_{\Lambda_{u}} e^{-\alpha L_{t}^{0}(s(X))}\right]}{\mathbb{E}_{s(x)}\left[e^{-\alpha L_{t}^{0}(s(X))}\right]} \\
& =\mathbb{P}_{s(x)}^{(\alpha, s(X))}\left(\Lambda_{u}\right) .
\end{aligned}
$$

Therefore, we shall always consider the equivalent probability under which $\left(X_{t}, t \geq 0\right)$ is a linear diffusion on natural scale.

3. The remainder of the paper is decomposed into five parts:

- In Section 2 , we prove Theorem 1.1, dealing only with the asymptotic of $\mathbb{E}\left[e^{-\alpha L_{t}}\right]$ $(\alpha>0)$. The proof relies on an analytic continuation of the Laplace transform of $t \mapsto \mathbb{E}\left[e^{-\alpha L_{t}}\right]$, and on the residue theorem.

- In Section 3, we prove Theorems 1.2 and 1.3 , still in the case of the penalization by $\left(e^{-\alpha L_{t}}, t \geq 0\right)$. The penalization by $\left(e^{\alpha L_{t}}, t \geq 0\right)$ being very similar, we shall only give, in Subsection 3.5, a few elements of the proof.

- In Section 4 , we prove Theorem 1.4 i.e. the iteration principle.

- In Section 5 , we derive explicit formulae when $X$ is a Brownian motion reflected at 0 and 1 , and more generally when $X$ is a Bessel process of dimension $\delta \in] 0,2[$ reflected at 1.

- And finally, in Section 6, we briefly deal with the cases of null recurrent and transient diffusions.

\section{$\S 2$. Proof of Theorem 1.1}

Let $\alpha>0$. We present the full proof of the penalization by $\left(e^{-\alpha L_{t}}, t \geq 0\right)$. A short proof of the penalization by $\left(e^{\alpha L_{t}}, t \geq 0\right)$ is given in Subsection 3.5. Let us recall that $X$ is a positively recurrent diffusion reflected on $[0, b]$ such that $b+m([0, b[)<\infty$. Our approach is based on the study of the Laplace transform of $t \mapsto \mathbb{E}_{x}\left[e^{-\alpha L_{t}}\right]$. Indeed, this quantity can be expressed explicitly in terms of the resolvent of $X$ :

Lemma 2.1. We have the identity

$$
\int_{0}^{\infty} e^{-\lambda t} \mathbb{E}_{x}\left[e^{-\alpha L_{t}}\right] d t=\frac{1}{\lambda}-\frac{R_{\lambda}(0, x)}{\lambda R_{\lambda}(0,0)} \frac{1}{1+\frac{1}{\alpha R_{\lambda}(0,0)}}
$$


Proof. Let $\lambda>0$. We have, from the Fubini-Tonelli theorem,

$$
\begin{aligned}
\int_{0}^{\infty} e^{-\lambda t} \mathbb{E}_{x}\left[e^{-\alpha L_{t}}\right] d t=\mathbb{E}_{x}\left[\int_{0}^{\infty} e^{-\lambda t} e^{-\alpha L_{t}} d t\right] \\
=\mathbb{E}_{x}\left[\frac{1}{\lambda}-\frac{\alpha}{\lambda} \int_{0}^{\infty} e^{-\lambda t} e^{-\alpha L_{t}} d L_{t}\right] \quad \text { after an integration by parts, } \\
=\frac{1}{\lambda}-\frac{\alpha}{\lambda} \int_{0}^{\infty} \mathbb{E}_{x}\left[e^{-\lambda \tau_{l}}\right] e^{-\alpha l} d l \quad \text { putting } L_{t}=l
\end{aligned}
$$

Since $X$ is a Markov process, $\tau$ is a subordinator and the following identities hold:

$$
\mathbb{E}_{x}\left[e^{-\lambda T_{0}}\right]=\frac{R_{\lambda}(0, x)}{R_{\lambda}(0,0)} \quad \text { and } \quad \mathbb{E}_{0}\left[e^{-\lambda \tau_{l}}\right]=\exp \left(-l / R_{\lambda}(0,0)\right),
$$

where $T_{0}:=\inf \left\{u \geq 0 ; X_{u}=0\right\}$ is the first hitting time of 0 by $X$. By the Markov property, 2.3 implies in particular that

$$
\mathbb{E}_{x}\left[e^{-\lambda \tau_{l}}\right]=\mathbb{E}_{x}\left[e^{-\lambda T_{0}}\right] \mathbb{E}_{0}\left[e^{-\lambda \tau_{l}}\right]=\frac{R_{\lambda}(0, x)}{R_{\lambda}(0,0)} \exp \left(-l / R_{\lambda}(0,0)\right) .
$$

Therefore, plugging 2.4 in 2.2, we get

$$
\begin{aligned}
\int_{0}^{\infty} e^{-\lambda t} \mathbb{E}_{x}\left[e^{-\alpha L_{t}}\right] d t & =\frac{1}{\lambda}-\frac{\alpha}{\lambda} \frac{R_{\lambda}(0, x)}{R_{\lambda}(0,0)} \int_{0}^{\infty} \exp \left(-l / R_{\lambda}(0,0)-\alpha l\right) d l \\
& =\frac{1}{\lambda}-\frac{\alpha}{\lambda} \frac{R_{\lambda}(0, x)}{R_{\lambda}(0,0)} \frac{1}{\alpha+\frac{1}{R_{\lambda}(0,0)}} \\
& =\frac{1}{\lambda}-\frac{R_{\lambda}(0, x)}{\lambda R_{\lambda}(0,0)} \frac{1}{1+\frac{1}{\alpha R_{\lambda}(0,0)}}
\end{aligned}
$$

We now determine the limit of $\int_{0}^{\infty} e^{-\lambda t} \mathbb{E}_{x}\left[e^{-\alpha L_{t}}\right] d t$ as $\lambda \rightarrow 0$. As shown by Lemma 2.1. we have to determine the rate of decay of $\lambda \mapsto R_{\lambda}(0,0)$ and $\lambda \mapsto R_{\lambda}(0, x)$.

Let us introduce the infinitesimal generator of $X$ :

$$
\mathcal{G}:=\frac{\partial^{2}}{\partial m \partial x}
$$

and, for $\lambda \in \mathbb{C}$, the two eigenfunctions $\Phi(\cdot, \lambda)$ and $\Psi(\cdot, \lambda)$, satisfying

$$
\left\{\begin{array} { l } 
{ \mathcal { G } [ \Phi ( \cdot , \lambda ) ] = \lambda \Phi ( \cdot , \lambda ) \text { on } [ 0 , b ] , } \\
{ \Phi ( 0 , \lambda ) = 1 \text { and } \Phi ^ { \prime } ( 0 , \lambda ) = 0 , }
\end{array} \text { and } \quad \left\{\begin{array}{l}
\mathcal{G}[\Psi(\cdot, \lambda)]=\lambda \Psi(\cdot, \lambda) \text { on }[0, b] \\
\Psi(0, \lambda)=0 \text { and } \Psi^{\prime}(0, \lambda)=1
\end{array}\right.\right.
$$


2.6 can be rewritten equivalently as

$$
\left\{\begin{array}{l}
\Phi(x, \lambda)=1+\lambda \int_{0}^{x} d y \int_{0}^{y} \Phi(s, \lambda) m(d s)=1+\lambda \int_{0}^{x}(x-s) \Phi(s, \lambda) m(d s), \\
\Psi(x, \lambda)=x+\lambda \int_{0}^{x} d y \int_{0}^{y} \Psi(s, \lambda) m(d s)=x+\lambda \int_{0}^{x}(x-s) \Psi(s, \lambda) m(d s),
\end{array}\right.
$$

where $x \in[0, b]$. Both $\Phi$ and $\Psi$ are entire functions in $\lambda$, differentiable in $x$ on $[0, b]$ since $m$ has no atoms, and positive if $\lambda$ is positive. According to DM76, Chapter V, p. 162], the resolvent kernel admits the representation

$$
R_{\lambda}(x, y)=\Phi(x, \lambda)\left(R_{\lambda}(0,0) \Phi(y, \lambda)-\Psi(y, \lambda)\right) \quad \text { for } x \leq y .
$$

Lemma 2.2. We have the following asymptotic behaviours:

$$
R_{\lambda}(0,0) \underset{\lambda \rightarrow 0}{\sim} \frac{1}{\lambda m([0, b])}
$$

and

$$
\frac{R_{\lambda}(0, x)}{R_{\lambda}(0,0)} \underset{\lambda \rightarrow 0}{=} 1+\lambda\left(\int_{0}^{x}(x-s) m(d s)-x m([0, b])\right)+o(\lambda) .
$$

Consequently,

$$
\int_{0}^{\infty} e^{-\lambda t} \mathbb{E}_{x}\left[e^{-\alpha L_{t}}\right] d t \underset{\lambda \rightarrow 0}{\sim} m([0, b])\left(x+\frac{1}{\alpha}\right)-\int_{0}^{x}(x-s) m(d s) .
$$

Proof. Since $b+m([0, b[)<\infty$ and $X$ is reflected at $b$, it is shown in [KK74, p. 34] that

$$
R_{\lambda}(0,0)=\frac{\Psi^{\prime}(b, \lambda)}{\Phi^{\prime}(b, \lambda)}
$$

Taking the $x$ derivative of 2.7 leads to

$$
R_{\lambda}(0,0)=\frac{1+\lambda \int_{0}^{b} \Psi(s, \lambda) m(d s)}{\lambda \int_{0}^{b} \Phi(s, \lambda) m(d s)}=\frac{1}{\lambda m([0, b])}+o\left(\frac{1}{\lambda}\right) \quad(\lambda \rightarrow 0) .
$$

Then identity (2.8) implies that

$$
\begin{aligned}
& \frac{R_{\lambda}(0, x)}{R_{\lambda}(0,0)}=\Phi(x, \lambda)-\frac{\Psi(x, \lambda)}{R_{\lambda}(0,0)} \\
& \quad=\Phi(x, \lambda)-\frac{x+\lambda \int_{0}^{x}(x-s) \Psi(s, \lambda) m(d s)}{\frac{1}{\lambda m([0, b])}+o(1 / \lambda)} \\
& \quad=\Phi(x, \lambda)-\lambda m([0, b])\left(x+\lambda \int_{0}^{x}(x-s) \Psi(s, \lambda) m(d s)\right)(1+o(1)) \\
& =1+\lambda\left(\int_{0}^{x}(x-s) m(d s)-x m([0, b])\right)+o(\lambda) .
\end{aligned}
$$


As a result, using (2.10), 2.11) and 2.1) we get

$$
\begin{aligned}
& \int_{0}^{\infty} e^{-\lambda t} \mathbb{E}_{x}\left[e^{-\alpha L_{t}}\right] d t \\
& \underset{\lambda \rightarrow 0}{=} \frac{1}{\lambda}\left(1-\left(1+\lambda\left(\int_{0}^{x}(x-s) m(d s)-x m([0, b])\right)+o(\lambda)\right) \frac{1}{1+\frac{\lambda m([0, b])}{\alpha}+o(\lambda)}\right) \\
& \underset{\lambda \rightarrow 0}{=} \frac{1}{\lambda}\left(1-\left(1+\lambda\left(\int_{0}^{x}(x-s) m(d s)-x m([0, b])\right)+o(\lambda)\right)\left(1-\frac{\lambda m([0, b])}{\alpha}+o(\lambda)\right)\right) \\
& \underset{\lambda \rightarrow 0}{\sim} m([0, b])\left(x+\frac{1}{\alpha}\right)-\int_{0}^{x}(x-s) m(d s) .
\end{aligned}
$$

Remark 2. Note that Lemma 2.2 implies that we cannot apply the Tauberian theorem (see Section 6 since the rate of decay of $\lambda \mapsto \int_{0}^{\infty} e^{-\lambda t} \mathbb{E}_{x}\left[e^{-\alpha L_{t}}\right] d t$ is not polynomial. Indeed, we will prove in Theorem 1.1 that it is in fact exponential.

Our approach now consists in extending (2.1) to $\lambda$ in the complex plane, in order to apply the inverse Fourier transform. To this end, we introduce some notation. We set $\mathbb{C}^{*}:=\mathbb{C} \backslash\{0\}, \mathbb{N}^{*}:=\mathbb{N} \backslash\{0\}$, and write $\mathbb{R}_{-}$(resp. $\mathbb{R}_{-}^{*}$ ) for the interval $]-\infty, 0]$ (resp. $]-\infty, 0[$ ). For a complex $z \in \mathbb{C}$, we denote by $\operatorname{Re}(z)$ the real part of $z$, and by $\operatorname{Im}(z)$ its imaginary part. Let us now define

$$
\mathcal{L}_{1}(z):=\int_{0}^{\infty} e^{-z t} \mathbb{E}_{x}\left[e^{-\alpha L_{t}}\right] d t .
$$

From Lemma 2.2, we see that $\mathcal{L}_{1}$ is well-defined on $\{z \in \mathbb{C} ; \operatorname{Re}(z) \geq 0\}$, and holomorphic on $\{z \in \mathbb{C} ; \operatorname{Re}(z)>0\}$. Let us introduce next

$$
f(s)= \begin{cases}0 & \text { if } s \leq-1, \\ s+1 & \text { if }-1 \leq s \leq 0, \\ \mathbb{E}_{x}\left[e^{-\alpha L_{s}}\right] & \text { if } s \geq 0\end{cases}
$$

and

$$
\mathcal{L}_{2}(z):=\int_{\mathbb{R}} e^{-z t} f(t) d t .
$$

Obviously

$$
\begin{aligned}
\mathcal{L}_{2}(z) & =\int_{-1}^{0} e^{-z t}(1+t) d t+\int_{0}^{\infty} e^{-z t} \mathbb{E}_{x}\left[e^{-\alpha L_{t}}\right] d t \\
& =-\frac{1}{z}-\frac{1-e^{z}}{z^{2}}+\mathcal{L}_{1}(z) .
\end{aligned}
$$

Consequently, $\mathcal{L}_{2}$ is once again well-defined on $\{z \in \mathbb{C} ; \operatorname{Re}(z) \geq 0\}$ and holomorphic on $\{z \in \mathbb{C} ; \operatorname{Re}(z)>0\}$. According to Lemma 2.2, $f$ belongs to $L^{1}(\mathbb{R})$, and 
therefore admits a Fourier transform:

$$
\widehat{f}(v):=\int_{\mathbb{R}} e^{i v t} f(t) d t=\mathcal{L}_{2}(-i v), \quad v \in \mathbb{R} .
$$

Our aim is to prove that $\widehat{f} \in L^{1}(\mathbb{R})$. This will permit inverting this transform. Let us start by rewriting $\mathcal{L}_{2}$ with the help of (2.1). Using $z=\lambda>0$ in 2.14 gives

$$
\mathcal{L}_{2}(\lambda)=-\frac{1-e^{\lambda}}{\lambda^{2}}-\frac{R_{\lambda}(0, x)}{\lambda R_{\lambda}(0,0)} \frac{\alpha}{\alpha+\frac{1}{R_{\lambda}(0,0)}} .
$$

Let us define

$$
H_{2}(z):=-\frac{1-e^{z}}{z^{2}}-\frac{R_{z}(0, x)}{z R_{z}(0,0)} \frac{\alpha}{\alpha+\frac{1}{R_{z}(0,0)}} .
$$

Lemma 2.3. $\mathrm{H}_{2}$ is a meromorphic function on $\mathbb{C}$, whose poles all belong to the negative real axis $\mathbb{R}_{-}^{*}$. We denote by $r^{2}$ the solution of the equation $1 / R_{-r^{2}}(0,0)$ $+\alpha=0$ of smallest modulus.

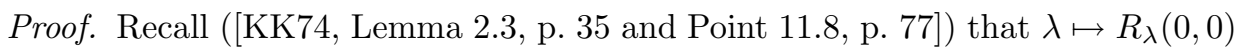
admits a meromorphic extension to $\mathbb{C}$, whose poles $\left(-\gamma_{n}^{2}\right)_{n \in \mathbb{N}}$ and zeros $\left(-\omega_{n}^{2}\right)_{n \in \mathbb{N}^{*}}$ are all negative. Then identity (2.8) implies that $\lambda \mapsto R_{\lambda}(0, x)$ also admits a meromorphic extension to $\mathbb{C}$, whose poles are $\left(-\gamma_{n}^{2}\right)_{n \in \mathbb{N}}$. Furthermore, from the identity ([KK74, Lemma 2.2, p. 34])

$$
\operatorname{Im}(\lambda) \int_{0}^{b}\left|\Phi(x, \lambda)-\frac{\Psi(x, \lambda)}{R_{\lambda}(0,0)}\right|^{2} m(d x)=\operatorname{Im}\left(R_{\lambda}(0,0)\right)
$$

we can conclude that $R_{\lambda}(0,0)$ is real if and only if $\lambda$ is real. But, when $\lambda>0$, it is clear from 1.1 that $R_{\lambda}(0,0)>0$. Therefore, the equation $1 / R_{z}(0,0)+\alpha=0$ can only have solutions in $\mathbb{R}_{-}$. Since $\int_{0}^{b} x m(d x)<+\infty$, it is known from DM76, Chapter V.6, p. 182] that

(i) $\gamma_{0}=0$,

(ii) the zeros $\left(-\omega_{n}^{2}\right)_{n \in \mathbb{N}^{*}}$ and the poles $\left(-\gamma_{n}^{2}\right)_{n \in \mathbb{N}}$ are interlaced,

(iii) for $\lambda \in \mathbb{R}$, the graph of $\lambda \mapsto 1 / R_{-\lambda^{2}}(0,0)$ is as in Figure 1 .

In particular, the equation $1 / R_{\lambda}(0,0)+\alpha=0$ admits a unique solution $\lambda=-r^{2}$ whose modulus is strictly smaller than $\omega_{1}^{2}$. Thus the function $z \mapsto \alpha /\left(\alpha+1 / R_{z}(0,0)\right)$ is meromorphic on $\mathbb{C}$ with all poles belonging to the negative real axis. Finally, it is clear that the part $z \mapsto-\left(1-e^{z}\right) / z^{2}$ is holomorphic on $\mathbb{C}^{*}$ and that 0 is not a pole of $H_{2}$ (from Lemma 2.2, , so we conclude that $H_{2}$ is a meromorphic function on $\mathbb{C}$ whose only pole in $\left\{z \in \mathbb{C} ; \operatorname{Re}(z)>-\omega_{1}^{2}\right\}$ is $-r^{2}$. 


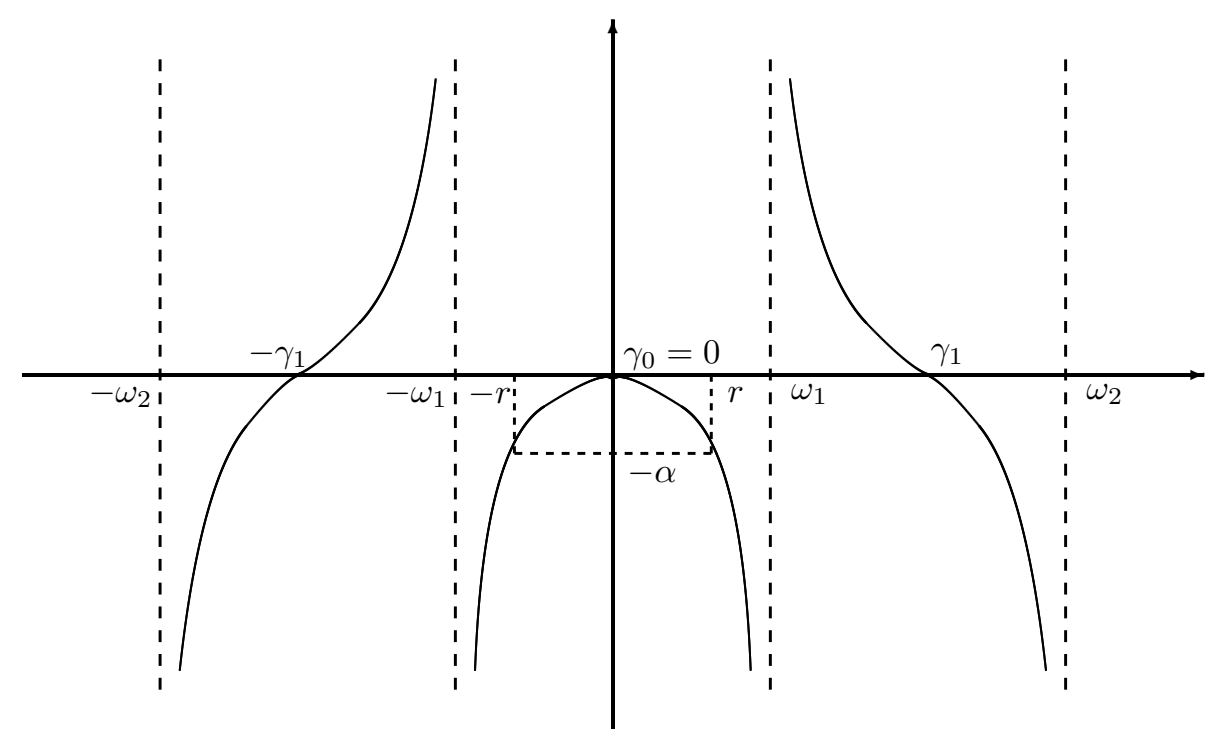

Fig. 1: Graph of $\lambda \mapsto 1 / R_{-\lambda^{2}}(0,0)$

Remark 3. An analytic continuation argument implies that the equality $\mathcal{L}_{2}(z)=$ $H_{2}(z)$ holds for all $z \in\{z \in \mathbb{C} ; \operatorname{Re}(z) \geq 0\}$. In particular, from 2.15), we have

$$
\widehat{f}(v)=\mathcal{L}_{2}(-i v)=H_{2}(-i v) \quad(v \in \mathbb{R}) .
$$

We now add the following technical assumption, which will ensure that $\widehat{f}$ is in $L^{1}(\mathbb{R})$ :

Assumption 2.1. We assume that there are $\beta>0$ and $c \in] r^{2}, \omega_{1}^{2}[$ such that, for $z \in\{z \in \mathbb{C} ;-c \leq \operatorname{Re}(z) \leq 0\}$,

$$
R_{z}(0,0) \underset{|z| \rightarrow+\infty}{=} \mathcal{O}\left(\frac{1}{|z|^{\beta}}\right) .
$$

This assumption is for instance satisfied by the Brownian motion reflected in $[0, b]$, and more generally by Bessel processes of dimension $\delta \in] 0,2[$ reflected at $b$ (see Section 5). It comes in useful in the following lemma:

Lemma 2.4. Let us assume that Assumption 2.1 holds. Then:

(i) For all $a \in[0, c] \backslash r^{2}$, the function $v \mapsto H_{2}(-a+i v)$ is integrable on $\mathbb{R}$, and tends to 0 when $v \rightarrow \pm \infty$.

(ii) $H_{2}$ is bounded on the domains $\{z \in \mathbb{C} ;-c \leq \operatorname{Re}(z) \leq 0, \operatorname{Im}(z) \geq 1\}$ and $\{z \in \mathbb{C} ;-c \leq \operatorname{Re}(z) \leq 0, \operatorname{Im}(z) \leq-1\}$. 
Proof. (i) First, it is clear from Lemma 2.3 that, in the domain $\{z \in \mathbb{C} ;-c \leq$ $\operatorname{Re}(z) \leq 0\}, H_{2}$ is a meromorphic function whose only pole is $-r^{2}$. Therefore, for $a \in[0, c] \backslash r^{2}$, the function $v \mapsto H_{2}(-a+i v)$ is continuous on $\mathbb{R}$, and we only have to check its integrability in the vicinity of $\pm \infty$. We have

$$
\begin{aligned}
H_{2}(-a+i v)= & -\frac{R_{-a+i v}(0, x)}{R_{-a+i v}(0,0)} \frac{\alpha}{(-a+i v)\left(\alpha+\frac{1}{R_{-a+i v}(0,0)}\right)} \\
& -\underbrace{\frac{1-e^{-a+i v}}{(-a+i v)^{2}}}_{\text {integrable at } \pm \infty} .
\end{aligned}
$$

On the one hand, using the first identity in 2.3 , we have

$$
\left|\frac{R_{-a+i v}(0, x)}{R_{-a+i v}(0,0)}\right|=\left|\mathbb{E}_{x}\left[e^{(a-i v) T_{0}}\right]\right| \leq \mathbb{E}_{b}\left[e^{c T_{0}}\right]<\infty .
$$

On the other hand, thanks to Assumption 2.1.

$$
\begin{aligned}
\frac{\alpha}{(-a+i v)\left(\alpha+\frac{1}{R_{-a+i v}(0,0)}\right)} & =\frac{R_{-a+i v}(0,0)}{(-a+i v)}\left(\frac{\alpha}{1+\alpha R_{-a+i v}(0,0)}\right) \\
& =\mathcal{O}\left(\frac{1}{|v|^{1+\beta}}\right) .
\end{aligned}
$$

Gathering (2.18) and (2.19), we obtain

$$
\frac{R_{-a+i v}(0, x)}{R_{-a+i v}(0,0)} \frac{\alpha}{(-a+i v)\left(\alpha+\frac{1}{R_{-a+i v}(0,0)}\right)} \underset{v \rightarrow \pm \infty}{=} \mathcal{O}\left(\frac{1}{|v|^{1+\beta}}\right) .
$$

Consequently, 2.17) and 2.20 imply that $v \mapsto H_{2}(-a+i v)$ belongs to $L^{1}(\mathbb{R})$.

(ii) More generally, 2.20 can be written, for $z \in\{z \in \mathbb{C} ;-c \leq \operatorname{Re}(z) \leq 0\}$, as

$$
\frac{R_{z}(0, x)}{R_{z}(0,0)} \frac{\alpha}{z\left(\alpha+\frac{1}{R_{z}(0,0)}\right)} \underset{|z| \rightarrow+\infty}{=} \mathcal{O}\left(\frac{1}{|z|^{1+\beta}}\right) .
$$

We only prove that $H_{2}$ is bounded on $\{z \in \mathbb{C} ;-c \leq \operatorname{Re}(z) \leq 0, \operatorname{Im}(z) \geq 1\}$. The same pattern of proof applies for the other case. Let $\varepsilon>0$. From 2.21, there exists $M>0$ such that, for all $z \in\{z \in \mathbb{C} ;-c \leq \operatorname{Re}(z) \leq 0\}$ satisfying $|z| \geq \operatorname{Im}(z) \geq M$, we have

$$
\left|H_{2}(z)\right|<\varepsilon .
$$

Therefore $\mathrm{H}_{2}$ is bounded on the domain $\{z \in \mathbb{C} ;-c \leq \operatorname{Re}(z) \leq 0, M \leq \operatorname{Im}(z)\}$. But, since $\mathrm{H}_{2}$ is continuous, it is also bounded on the compact domain $\{z \in \mathbb{C}$; $-c \leq \operatorname{Re}(z) \leq 0,1 \leq \operatorname{Im}(z) \leq M\}$. This ends the proof of Lemma 2.4. 
In particular, for $a=0$, we infer that $\widehat{f} \in L^{1}(\mathbb{R})$. We can therefore apply the inverse Fourier transform to get

$$
f(t)=\frac{1}{2 \pi} \int_{\mathbb{R}} e^{-i v t} \widehat{f}(v) d v=\frac{1}{2 \pi} \int_{\mathbb{R}} e^{-i v t} H_{2}(-i v) d v .
$$

To obtain an equivalent to $f(t)$ when $t$ tends to infinity, we consider the integration contour $\Delta_{R}=\Delta_{R}^{1} \cup \Delta_{R}^{2} \cup \Delta_{R}^{3} \cup \Delta_{R}^{4}$ of Figure 2, on which we will apply the residue theorem to the meromorphic function $z \mapsto e^{t z} H_{2}(z)$.

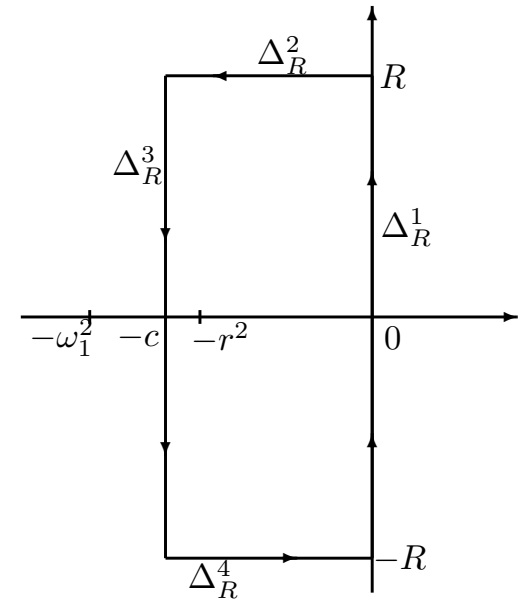

Fig. 2: Integration contour

Lemma 2.5. Let $t>0$ be fixed and $r^{2}<c<\omega_{1}^{2}$.

(i) We have

$$
\oint_{\Delta_{R}} e^{t z} H_{2}(z) d z \underset{R \rightarrow+\infty}{\longrightarrow} 2 i \pi f(t)+\int_{\Delta_{\infty}^{3}} e^{t z} H_{2}(z) d z
$$

where $\Delta_{\infty}^{3}$ is the axis $-c+i \mathbb{R}$.

(ii) There is a constant $K(x)$ independent of $t$ such that

$$
\left|\int_{\Delta_{\infty}^{3}} e^{t z} H_{2}(z) d z\right| \leq K(x) e^{-c t}
$$

Proof. We study each side of the rectangle separately:

1) We parametrize $\Delta_{R}^{1}$ with $z=i v,-R \leq v \leq R$. Then, from 2.22,

$$
\begin{aligned}
\int_{\Delta_{R}^{1}} e^{t z} H_{2}(z) d z & =i \int_{-R}^{R} e^{i t v} H_{2}(i v) d v \\
& =i \int_{-R}^{R} e^{-i t v} H_{2}(-i v) d v \underset{R \rightarrow+\infty}{\longrightarrow} 2 i \pi f(t) .
\end{aligned}
$$


2) Let $\{z=-a+i R ; 0 \leq a \leq c\}$ be a parametrization of $\Delta_{R}^{2}$. Then

$$
\int_{\Delta_{R}^{2}} e^{t z} H_{2}(z) d z=\int_{0}^{c} e^{t(-a+i R)} H_{2}(-a+i R) d a .
$$

According to Lemma 2.4 the function $z \mapsto H_{2}(z)$ is bounded on $\{z \in \mathbb{C}$; $-c \leq$ $\operatorname{Re}(z) \leq 0, \operatorname{Im}(z) \geq 1\}$, and $\lim _{R \rightarrow+\infty} H_{2}(-a+i R)=0$. Then we can apply the dominated convergence theorem to obtain

$$
\lim _{R \rightarrow+\infty} \int_{\Delta_{R}^{2}} e^{t z} H_{2}(z) d z=0 .
$$

3) We parametrize $\Delta_{R}^{4}$ with $z=-a-i R, 0 \leq a \leq c$. The proof on this segment is the same as the one on $\Delta_{R}^{2}$, so we get

$$
\lim _{R \rightarrow+\infty} \int_{\Delta_{R}^{4}} e^{t z} H_{2}(z) d z=0 .
$$

4) As for $\Delta_{R}^{3}$, we use $z=-c-i v,-R \leq v \leq R$, to obtain

$$
\left|\int_{\Delta_{R}^{3}} e^{t z} H_{2}(z) d z\right|=\left|\int_{R}^{-R} e^{-c t-i v t} H_{2}(-c-i v) i d v\right| \leq e^{-c t} K(x),
$$

where $K(x)=\int_{-\infty}^{\infty}\left|H_{2}(-c+i v)\right| d v$. From Lemma 2.4. $K(x)$ is finite. This shows (2.24. Moreover,

$$
\lim _{R \rightarrow+\infty} \int_{\Delta_{R}^{3}} e^{t z} H_{2}(z) d z=\int_{\Delta_{\infty}^{3}} e^{t z} H_{2}(z) d z .
$$

It is then clear that 2.23 is a direct consequence of 2.25-2.28.

Proof of Theorem 1.1. From 2.16, we have

$$
e^{t z} H_{2}(z)=-e^{t z} \frac{R_{z}(0, x)}{z R_{z}(0,0)} \frac{\alpha}{\alpha+\frac{1}{R_{z}(0,0)}}-e^{t z} \frac{1-e^{z}}{z^{2}} .
$$

The only pole of $z \mapsto e^{t z} H_{2}(z)$ inside the contour $\Delta_{R}$ is $-r^{2}$, and it is a simple one. The part $e^{t z}\left(1-e^{z}\right) / z^{2}$ has no contribution since it is holomorphic at $-r^{2}$. Consequently, the residue of $e^{t z} H_{2}(z)$ at $-r^{2}$ reduces to

$$
\begin{aligned}
\operatorname{Res}\left(e^{t z} H_{2}(z),-r^{2}\right) & =\frac{R_{-r^{2}}(0, x)}{r^{2} R_{-r^{2}}(0,0)} \frac{\alpha}{\left.\frac{\partial}{\partial z}\left(\alpha+\frac{1}{R_{z}(0,0)}\right)\right|_{z=-r^{2}}} \exp \left(-r^{2} t\right) \\
& =\frac{1}{r^{2}} R_{-r^{2}}(0, x) \frac{1}{\left.\frac{\partial}{\partial z} R_{z}(0,0)\right|_{z=-r^{2}}} \exp \left(-r^{2} t\right)
\end{aligned}
$$


Applying the residue theorem and 2.23 leads to

$$
2 i \pi f(t)+\int_{\Delta_{\infty}^{3}} e^{t z} H_{2}(z) d z=\frac{2 i \pi}{r^{2}} R_{-r^{2}}(0, x) \frac{1}{\left.\frac{\partial}{\partial z} R_{z}(0,0)\right|_{z=-r^{2}}} \exp \left(-r^{2} t\right) .
$$

Since $c>r$, using 2.24 we get

$$
f(t)=\mathbb{E}_{x}\left[e^{-\alpha L_{t}}\right] \underset{t \rightarrow+\infty}{\sim} \frac{1}{r^{2}} R_{-r^{2}}(0, x) \frac{1}{\left.\frac{\partial}{\partial z} R_{z}(0,0)\right|_{z=-r^{2}}} \exp \left(-r^{2} t\right),
$$

which ends the proof of Theorem 1.1

\section{§3. Proofs of Theorems 1.2 and 1.3}

As in the previous section, we shall only deal with the case of the penalization by $\left(e^{-\alpha L_{t}}, t \geq 0\right)$. Some comments about the case $\left(e^{\alpha L_{t}}, t \geq 0\right)$ will be given in Subsection 3.5. We assume from now on that $m$ is absolutely continuous with respect to the Lebesgue measure: $m(d x)=m(x) d x$.

\section{§3.1. A preliminary lemma}

Lemma 3.1. Let $\alpha>0$, and $r^{2}$ be the unique solution in $] 0, \omega_{1}^{2}[$ of the equation $\alpha+1 / R_{-r^{2}}(0,0)=0$. Then, for $x \in[0, b]$, the process

$$
\left(M_{t}^{(-\alpha)}:=\exp \left(r^{2} t-\alpha L_{t}\right) \frac{R_{-r^{2}}\left(0, X_{t}\right)}{R_{-r^{2}}(0, x)}, t \geq 0\right)
$$

is a continuous, strictly positive $\mathbb{P}_{x}$-martingale which converges to 0 as $t \rightarrow+\infty$.

Proof. 1) Relation (2.8) implies that

$$
\frac{R_{\lambda}(0, x)}{R_{\lambda}(0,0)}=\Phi(x, \lambda)-\frac{\Psi(x, \lambda)}{R_{\lambda}(0,0)} .
$$

We have noticed in the proof of Lemma 2.3 that $z \mapsto 1 / R_{z}(0,0)$ is holomorphic on the domain $\left\{z \in \mathbb{C} ; \operatorname{Re}(z)>-\omega_{1}^{2}\right\}$. An analytic continuation argument applied to the first identity in 2.3 leads to

$$
\mathbb{E}_{x}\left[e^{r^{2} T_{0}}\right]=\frac{R_{-r^{2}}(0, x)}{R_{-r^{2}}(0,0)}<\infty .
$$

This implies that $M^{(-\alpha)}$ is continuous and strictly positive. We now assume that $x=0$ to simplify the notations. According to RW00, Chapter V, Theorem 47.1, p. 277], there exists a Brownian motion $\left(B_{t}, t \geq 0\right)$ reflected at 0 and $b$ such that

$$
X_{t}=B_{\gamma_{t}} \quad(t \geq 0),
$$

where: 
- $\left(L_{t}^{z}(B), z \in[0, b], t \geq 0\right)$ is the local time at $z$ of the process $\left(B_{t}, t \geq 0\right)$,

- $A_{t}=\int_{0}^{b} L_{t}^{z}(B) m(d z)$ is a continuous additive functional,

- $\gamma_{t}=\inf \left\{u \geq 0 ; A_{u}>t\right\}$ is the right-continuous inverse of $A$.

Note that $L_{t}^{z}(X)=L_{\gamma_{t}}^{z}(B)$. Here, since we have assumed that $m$ has a density, we have, from the occupation time formula,

$$
A_{t}=\int_{0}^{b} L_{t}^{z}(B) m(z) d z=\frac{1}{2} \int_{0}^{t} m\left(B_{s}\right) d s .
$$

As a result, $A$ is continuous and strictly increasing, so that $\gamma$ is also continuous, strictly increasing, and $A_{\gamma_{t}}=\gamma_{A_{t}}=t$.

2) Let us apply Itô's formula. In the following, all the derivatives are taken with respect to the first variable, for example $\Phi^{\prime}(x, \lambda):=\frac{\partial \Phi}{\partial x}(x, \lambda)$. We have

$$
\begin{aligned}
e^{r^{2} A_{t}-\alpha L_{t}(B)}\left(\frac{R_{-r^{2}}\left(0, B_{t}\right)}{R_{-r^{2}}(0,0)}\right)=e^{r^{2} A_{t}-\alpha L_{t}(B)}\left(\Phi\left(B_{t},-r^{2}\right)-\frac{\Psi\left(B_{t},-r^{2}\right)}{R_{-r^{2}}(0,0)}\right) \\
=1+\int_{0}^{t} e^{r^{2} A_{s}-\alpha L_{s}(B)}\left(\Phi^{\prime}\left(B_{s},-r^{2}\right)-\frac{\Psi^{\prime}\left(B_{s},-r^{2}\right)}{R_{-r^{2}}(0,0)}\right) d B_{s} \\
\quad+\frac{1}{2} \int_{0}^{t} e^{r^{2} A_{s}-\alpha L_{s}(B)}\left(\Phi^{\prime \prime}\left(B_{s},-r^{2}\right)-\frac{\Psi^{\prime \prime}\left(B_{s},-r^{2}\right)}{R_{-r^{2}}(0,0)}\right) d s \\
\quad+r^{2} \int_{0}^{t} e^{r^{2} A_{s}-\alpha L_{s}(B)}\left(\Phi\left(B_{s},-r^{2}\right)-\frac{\Psi\left(B_{s},-r^{2}\right)}{R_{-r^{2}}(0,0)}\right) d A_{s} \\
\quad-\alpha \int_{0}^{t} e^{r^{2} A_{s}-\alpha L_{s}(B)} d L_{s}(B) .
\end{aligned}
$$

We then replace $t$ by $\gamma_{t}$ and make the time change $s=\gamma_{u}$, following Proposition 1.5, p. 181, from D. Revuz and M. Yor [RY99]. This entails

$$
\begin{aligned}
M_{t}^{(-\alpha)}=1 & +\int_{0}^{t} e^{r^{2} u-\alpha L_{u}(X)}\left(\Phi^{\prime}\left(X_{u},-r^{2}\right)-\frac{\Psi^{\prime}\left(X_{u},-r^{2}\right)}{R_{-r^{2}}(0,0)}\right) d X_{u} \\
& -\frac{r^{2}}{2} \int_{0}^{t} e^{r^{2} u-\alpha L_{u}(X)}\left(\Phi\left(X_{u},-r^{2}\right)-\frac{\Psi\left(X_{u},-r^{2}\right)}{R_{-r^{2}}(0,0)}\right) m\left(X_{u}\right) d \gamma_{u} \\
& +r^{2} \int_{0}^{t} e^{r^{2} u-\alpha L_{u}(X)}\left(\Phi\left(X_{u},-r^{2}\right)-\frac{\Psi\left(X_{u},-r^{2}\right)}{R_{-r^{2}}(0,0)}\right) d u \\
& -\alpha \int_{0}^{t} e^{r^{2} u-\alpha L_{u}(X)} d L_{u}(X),
\end{aligned}
$$

where, in (3.4), we have used the fact that $\Phi$ and $\Psi$ are eigenfunctions of the operator $\mathcal{G}$ (cf. (2.6)). Then differentiating the equality $A_{\gamma_{t}}=t$, we get from (3.2)

$$
d \gamma_{u}=\frac{2}{m\left(X_{u}\right)} d u
$$


As a result, the terms related to $d u((3.4)$ and $(3.5)$ ) cancel. Let us now examine the coefficients with respect to $d L_{t}(X)$ and $d L_{t}^{b}(X)$. Since $B$ can be written as $\beta+L^{0}(B)-L^{b}(B)$ where $\beta$ is a standard Brownian motion, we have, by time change,

$$
X=\beta_{\gamma}+L^{0}(X)-L^{b}(X)
$$

where $\beta_{\gamma}$ is an $\left(\mathcal{F}_{\gamma_{t}}\right)$-local martingale.

(i) From 2.6), $\Phi^{\prime}\left(0,-r^{2}\right)=0$ and $\Psi^{\prime}\left(0,-r^{2}\right)=1$. Then 3.3 and 3.6 give

$$
\left.-\left(\frac{1}{R_{-r^{2}}(0,0)}+\alpha\right) d L_{s}^{0}(X)=0 \quad \text { by definition of } r \text { (cf. } 1.2\right) \text {. }
$$

(ii) 3.3 gives

$$
\left.\left(\Phi^{\prime}\left(b,-r^{2}\right)-\frac{\Psi^{\prime}\left(b,-r^{2}\right)}{R_{-r^{2}}(0,0)}\right) d L_{s}^{b}(X)=0 \quad \text { by definition of } R_{-r^{2}}(0,0) \text { (cf. } 2.9 p\right) \text {. }
$$

Finally, 3.3) reduces to

$$
M_{t}^{(-\alpha)}=1+\int_{0}^{t} e^{r^{2} u-\alpha L_{u}}\left(\Phi^{\prime}\left(X_{u},-r^{2}\right)-\frac{\Psi^{\prime}\left(X_{u},-r^{2}\right)}{R_{-r^{2}}(0,0)}\right) d \beta_{\gamma_{u}} .
$$

This implies that $M^{(-\alpha)}$ is a continuous local martingale. But, from 3.1 , we have

$$
\begin{aligned}
M_{t}^{(-\alpha)} & =e^{r^{2} t-\alpha L_{t}} \frac{R_{-r^{2}}\left(0, X_{t}\right)}{R_{-r^{2}}(0,0)} \\
& \leq e^{r^{2} t} \mathbb{E}_{X_{t}}\left[e^{r^{2} T_{0}}\right] \\
& \leq e^{r^{2} t} \mathbb{E}_{b}\left[e^{r^{2} T_{0}}\right] \quad \text { since } x \mapsto \mathbb{E}_{x}\left[e^{r^{2} T_{0}}\right] \text { is clearly increasing. }
\end{aligned}
$$

As a result, $M^{(-\alpha)}$ is a positive $\mathbb{P}$-martingale, and therefore converges almost surely.

3) Using (3.1), let us write

$$
\begin{aligned}
M_{t}^{(-\alpha)} & =e^{r^{2} t-\alpha L_{t}} \frac{R_{-r^{2}}\left(0, X_{t}\right)}{R_{-r^{2}}(0,0)} \\
& \leq \exp \left(-\alpha L_{t}\left(1-\frac{r^{2} t}{\alpha L_{t}}\right)\right) \mathbb{E}_{b}\left[e^{r^{2} T_{0}}\right] .
\end{aligned}
$$

From an ergodic theorem (see [IM74, Chapter 6, p. 229]), we know that

$$
\frac{L_{t}}{t} \underset{t \rightarrow+\infty}{\longrightarrow} \frac{1}{m([0, b])} \quad \text { a.s. }
$$

Let us apply Jensen's inequality with the convex functions $x \mapsto x^{k}(k \in \mathbb{N})$ :

$$
\frac{\left(r^{2} \mathbb{E}_{0}\left[\tau_{l}\right]\right)^{k}}{k !} \leq \frac{\mathbb{E}_{0}\left[\left(r^{2} \tau_{l}\right)^{k}\right]}{k !} \quad(l>0)
$$


With $k=2$, it is clear from the equality case in the Cauchy-Schwarz inequality that

$$
\left(r^{2} \mathbb{E}_{0}\left[\tau_{l}\right]\right)^{2}<\mathbb{E}_{0}\left[\left(r^{2} \tau_{l}\right)^{2}\right] .
$$

Therefore, summing (3.9) with respect to $k$, we obtain

$$
\exp \left(\mathbb{E}_{0}\left[r^{2} \tau_{l}\right]\right)<\mathbb{E}_{0}\left[\exp \left(r^{2} \tau_{l}\right)\right]
$$

and this inequality is strict. Now, it is known from [BS02, p. 22] that

$$
\mathbb{E}_{0}\left[\tau_{l}\right]=m([0, b]) l .
$$

Hence, plugging (3.11) and 2.3) (with $\lambda=-r^{2}$ ) in 3.10), we get

$$
e^{r^{2} m([0, b]) l}<e^{-l / R_{-r^{2}}(0,0)} \Leftrightarrow-r^{2} R_{-r^{2}}(0,0) m([0, b])<1,
$$

since $R_{-r^{2}}(0,0)=-1 / \alpha<0$. Consequently, using 3.8 and 1.2 , we obtain

(3.12) $\lim _{t \rightarrow+\infty}\left(1-\frac{r^{2} t}{\alpha L_{t}}\right)=1-\frac{r^{2} m([0, b])}{\alpha}=1+r^{2} R_{-r^{2}}(0,0) m([0, b])>0 \quad$ a.s.

Finally, letting $t \rightarrow+\infty$ in 3.7 and using 3.12 ends the proof of Lemma 3.1 .

\section{§3.2. Proof of Theorem 1.2}

We will use the following general penalization principle (see [RVY06]):

Theorem 3.1. Let $\left(\Gamma_{t}, t \geq 0\right)$ be a stochastic process satisfying, for every $t \geq 0$, $0<\mathbb{E}\left[\Gamma_{t}\right]<+\infty$. Suppose that, for any $s \geq 0$,

$$
\lim _{t \rightarrow+\infty} \frac{\mathbb{E}\left[\Gamma_{t} \mid \mathcal{F}_{s}\right]}{\mathbb{E}\left[\Gamma_{t}\right]}=: M_{s}
$$

exists a.s., and

$$
\mathbb{E}\left[M_{s}\right]=1
$$

Then:

(i) For any $s \geq 0$ and $\Lambda_{s} \in \mathcal{F}_{s}$,

$$
\lim _{t \rightarrow+\infty} \frac{\mathbb{E}\left[1_{\Lambda_{s}} \Gamma_{t}\right]}{\mathbb{E}\left[\Gamma_{t}\right]}=\mathbb{E}\left[M_{s} 1_{\Lambda_{s}}\right] .
$$

(ii) There exists a probability measure $\mathbb{Q}$ on $\left(\Omega, \mathcal{F}_{\infty}\right)$ such that for any $s>0$,

$$
\mathbb{Q}\left(\Lambda_{s}\right)=\mathbb{E}\left[M_{s} 1_{\Lambda_{s}}\right] .
$$


In our framework, we have, for $s<t$, by the Markov property and Theorem 1.1 .

$$
\begin{aligned}
\frac{\mathbb{E}_{x}\left[e^{-\alpha L_{t}} \mid \mathcal{F}_{s}\right]}{\mathbb{E}_{x}\left[e^{-\alpha L_{t}}\right]}=\frac{e^{-\alpha L_{s}} \mathbb{E}_{X_{s}}\left[e^{-\alpha L_{t-s}}\right]}{\mathbb{E}_{x}\left[e^{-\alpha L_{t}}\right]} & \underset{t \rightarrow+\infty}{\longrightarrow} \exp \left(r^{2} s-\alpha L_{s}\right) \frac{R_{-r^{2}}\left(0, X_{s}\right)}{R_{-r^{2}}(0, x)}=M_{s}^{(-\alpha)} .
\end{aligned}
$$

Note that from Lemma 3.1. $M^{(-\alpha)}$ is a martingale such that $\mathbb{E}_{x}\left[M_{s}^{(-\alpha)}\right]=1$. This proves Theorem 1.2 .

\section{$\S 3.3$. Proof of Theorem 1.3}

Proof of Theorem 1.3 (i). 1) We start by proving that the coordinate process $X$ is still a Markov process under $\mathbb{P}_{x}^{(-\alpha)}$. Let $\Lambda_{s} \in \mathcal{F}_{s}$, and $f$ be a Borel function with compact support. We have, for $s \leq t$,

$$
\begin{aligned}
\mathbb{E}_{x}^{(-\alpha)}\left[f\left(X_{t+s}\right) 1_{\Lambda_{s}}\right] & =\mathbb{E}_{x}\left[M_{t+s}^{(-\alpha)} f\left(X_{t+s}\right) 1_{\Lambda_{s}}\right] \\
= & \mathbb{E}_{x}\left[\exp \left(r^{2}(t+s)-\alpha L_{t+s}\right) \frac{R_{-r^{2}}\left(0, X_{t+s}\right)}{R_{-r^{2}}(0, x)} f\left(X_{t+s}\right) 1_{\Lambda_{s}}\right] \\
= & \mathbb{E}_{x}\left[\frac{e^{r^{2}(t+s)}}{R_{-r^{2}}(0, x)} \mathbb{E}_{x}\left[e^{-\alpha L_{t+s}} R_{-r^{2}}\left(0, X_{t+s}\right) f\left(X_{t+s}\right) \mid \mathcal{F}_{s}\right] 1_{\Lambda_{s}}\right] \\
= & \mathbb{E}_{x}\left[\frac{e^{r^{2}(t+s)-\alpha L_{s}}}{R_{-r^{2}}(0, x)} \mathbb{E}_{x}\left[e^{-\alpha L_{t} \circ \theta_{s}} R_{-r^{2}}\left(0, X_{t} \circ \theta_{s}\right) f\left(X_{t} \circ \theta_{s}\right) \mid \mathcal{F}_{s}\right] 1_{\Lambda_{s}}\right] \\
& =\mathbb{E}_{x}\left[\frac{e^{r^{2}(t+s)-\alpha L_{s}}}{R_{-r^{2}}(0, x)} \mathbb{E}_{X_{s}}\left[e^{-\alpha L_{t}} R_{-r^{2}}\left(0, X_{t}\right) f\left(X_{t}\right)\right] 1_{\Lambda_{s}}\right] \\
& =\mathbb{E}_{x}\left[e^{r^{2} s-\alpha L_{s}} \frac{R-r^{2}\left(0, X_{s}\right)}{R_{-r^{2}}(0, x)} \mathbb{E}_{X_{s}}^{(-\alpha)}\left[f\left(X_{t}\right)\right] 1_{\Lambda_{s}}\right] \\
& =\mathbb{E}_{x}^{(-\alpha)}\left[\mathbb{E}_{X_{s}}^{(-\alpha)}\left[f\left(X_{t}\right)\right] 1_{\Lambda_{s}}\right] .
\end{aligned}
$$

Therefore, we obtain

$$
\mathbb{E}_{x}^{(-\alpha)}\left[f\left(X_{t+s}\right) \mid \mathcal{F}_{s}\right]=\mathbb{E}_{X_{s}}^{(-\alpha)}\left[f\left(X_{t}\right)\right]
$$

This proves that $X$ is Markov under $\mathbb{P}_{x}^{(-\alpha)}$.

2) Let us calculate its infinitesimal generator. Let $f$ be a bounded function defined on $\mathbb{R}_{+}$, and of class $\mathcal{C}^{2}$. Then

$$
\frac{1}{t} \mathbb{E}_{x}^{(-\alpha)}\left[f\left(X_{t}\right)-f(x)\right]=\frac{1}{t} \mathbb{E}_{x}\left[\left(f\left(X_{t}\right)-f(x)\right) \frac{R_{-r^{2}}\left(0, X_{t}\right)}{R_{-r^{2}}(0, x)} e^{r^{2} t-\alpha L_{t}}\right]
$$




$$
\begin{aligned}
= & \frac{1}{t} \frac{1}{R_{-r^{2}}(0, x)}\left(\mathbb{E}_{x}\left[\left(f\left(X_{t}\right) R_{-r^{2}}\left(0, X_{t}\right)-f(x) R_{-r^{2}}(0, x)\right) e^{r^{2} t-\alpha L_{t}}\right]\right. \\
& \left.\quad-f(x) \mathbb{E}_{x}\left[\left(R_{-r^{2}}\left(0, X_{t}\right)-R_{-r^{2}}(0, x)\right) e^{r^{2} t-\alpha L_{t}}\right]\right) \\
\underset{t \rightarrow+\infty}{\longrightarrow} & \frac{1}{R_{-r^{2}}(0, x)}\left(\mathcal{G}\left(R_{-r^{2}}(0, \cdot) f\right)(x)-f(x) \mathcal{G}\left(R_{-r^{2}}(0, \cdot)\right)\right) \\
= & \frac{1}{R_{-r^{2}}(0, x)} \mathcal{G}\left(R_{-r^{2}}(0, \cdot) f\right)(x)+r^{2} f(x),
\end{aligned}
$$

since $x \mapsto R_{-r^{2}}(0, x)$ is an eigenfunction of the operator $\mathcal{G}$ associated to the eigenvalue $-r^{2}$. Using the definition of $\mathcal{G}$ (cf. (2.5)), we finally get

$$
\mathcal{G}^{(-\alpha)} f(x)=\frac{1}{m(x)} f^{\prime \prime}(x)+\frac{2}{m(x) R_{-r^{2}}(0, x)} \frac{\partial R_{-r^{2}}(0, x)}{\partial x} f^{\prime}(x) .
$$

3) Let us determine the domain of $\mathcal{G}^{(-\alpha)}$. Applying [RY99, Exercise 3.20, p. 311] to the expression 3.13, we see that the scale function of $X$ under $\mathbb{P}^{(-\alpha)}$ equals

$$
s^{(-\alpha)}(x)=\int_{0}^{x}\left(\frac{R_{-r^{2}}(0,0)}{R_{-r^{2}}(0, y)}\right)^{2} d y=\int_{0}^{x} \frac{d y}{\left(\mathbb{E}_{y}\left[e^{r^{2} T_{0}}\right]\right)^{2}},
$$

and the speed measure $m^{(-\alpha)}$ is

$$
m^{(-\alpha)}(x)=\left(\frac{R_{-r^{2}}(0, x)}{R_{-r^{2}}(0,0)}\right)^{2} m(x)=\left(\mathbb{E}_{x}\left[e^{r^{2} T_{0}}\right]\right)^{2} m(x) .
$$

Then, for $z \in] 0, b\left[\right.$, since $1 \leq \mathbb{E}_{x}\left[e^{r^{2} T_{0}}\right] \leq \mathbb{E}_{b}\left[e^{r^{2} T_{0}}\right]$, it is clear that

$$
\left\{\begin{array}{l}
\int_{0}^{z}\left(\int_{y}^{z}\left(\mathbb{E}_{x}\left[e^{r^{2} T_{0}}\right]\right)^{2} m(x) d x\right) \frac{d y}{\left(\mathbb{E}_{y}\left[e^{r^{2} T_{0}}\right]\right)^{2}} \leq b m([0, b])\left(\mathbb{E}_{b}\left[e^{r^{2} T_{0}}\right]\right)^{2}<\infty, \\
\int_{0}^{z}\left(\int_{y}^{z} \frac{d x}{\left(\mathbb{E}_{x}\left[e^{r^{2} T_{0}}\right]\right)^{2}}\right) \mathbb{E}_{y}\left(\left[e^{r^{2} T_{0}}\right]\right)^{2} m(y) d y \leq b m([0, b])\left(\mathbb{E}_{b}\left[e^{r^{2} T_{0}}\right]\right)^{2}<\infty,
\end{array}\right.
$$

which means that 0 is a non-singular boundary (see BS02, p. 14]). Since $m^{(-\alpha)}$ admits a density, we have $m^{(-\alpha)}(\{0\})=0$ and 0 is a reflecting boundary. The same is true for the endpoint $b$, and

$$
\mathcal{D}\left(\mathcal{G}^{(-\alpha)}\right):=\left\{f: \mathcal{G}^{(-\alpha)} f \in \mathcal{C}_{\mathrm{b}}([0, b]), f^{\prime}\left(0^{+}\right)=f^{\prime}\left(b^{-}\right)=0\right\} .
$$

Proof of Theorem 1.3(ii). Let us introduce the density $n$ of the Lévy measure of $\tau$ under $\mathbb{P}$. Since $\tau$ is a subordinator, it is known, using $(2.3)$, that

$$
\frac{1}{R_{\lambda}(0,0)}=\int_{0}^{\infty}\left(1-e^{-\lambda u}\right) n(u) d u \text {. }
$$


To obtain the Lévy measure of $\tau$ under $\mathbb{P}^{(-\alpha)}$, we start by computing its Laplace transform. Since under $\mathbb{P}_{x}$,

$$
M_{\tau_{l}}^{(-\alpha)}=\frac{R_{-r^{2}}(0,0)}{R_{-r^{2}}(0, x)} \exp \left(r^{2} \tau_{l}-\alpha l\right)
$$

Doob's Optional Stopping Theorem gives, for $\lambda \geq 0$,

$$
\mathbb{E}_{x}^{(-\alpha)}\left[e^{-\lambda \tau_{l}} 1_{\left\{\tau_{l} \leq t\right\}}\right]=e^{-\alpha l} \frac{R_{-r^{2}}(0,0)}{R_{-r^{2}}(0, x)} \mathbb{E}_{x}\left[e^{-\left(\lambda-r^{2}\right) \tau_{l}} 1_{\left\{\tau_{l} \leq t\right\}}\right] .
$$

Then, letting $t \rightarrow+\infty$ in 3.17 and applying the monotone convergence theorem, we get

$$
\begin{aligned}
\mathbb{E}_{x}^{(-\alpha)}\left[e^{-\lambda \tau_{l}}\right] & =\frac{R_{-r^{2}}(0,0)}{R_{-r^{2}}(0, x)} e^{-\alpha l} \mathbb{E}_{x}\left[e^{-\left(\lambda-r^{2}\right) \tau_{l}}\right] \\
& =\frac{R_{-r^{2}}(0,0)}{R_{-r^{2}}(0, x)} \frac{R_{\lambda-r^{2}}(0, x)}{R_{\lambda-r^{2}}(0,0)} e^{-l\left(\alpha+1 / R_{\lambda-r^{2}}(0,0)\right)} \quad(\text { from } 2.4)
\end{aligned}
$$

Now, formula 3.16 yields

$$
\begin{aligned}
\alpha+\frac{1}{R_{\lambda-r^{2}}(0,0)} & =\alpha+\int_{0}^{\infty}\left(1-e^{-\left(\lambda-r^{2}\right) u}\right) n(u) d u \\
& =\alpha+\int_{0}^{\infty}\left(1-e^{r^{2} u}\right) n(u) d u+\int_{0}^{\infty}\left(e^{r^{2} u}-e^{-\left(\lambda-r^{2}\right) u}\right) n(u) d u \\
& =\alpha+\frac{1}{R_{-r^{2}}(0,0)}+\int_{0}^{\infty}\left(1-e^{-\lambda u}\right) e^{r^{2} u} n(u) d u \\
& =\int_{0}^{\infty}\left(1-e^{-\lambda u}\right) e^{r^{2} u} n(u) d u \quad \text { since } \alpha+\frac{1}{R_{-r^{2}}(0,0)}=0,
\end{aligned}
$$

which shows (ii).

Proof of Theorem 1.3 (iii). To evaluate $\mathbb{P}_{x}^{(-\alpha)}\left(L_{t} \geq l\right)$, we rewrite 3.18 with $\lambda=0$ :

$$
\begin{aligned}
\mathbb{P}_{x}^{(-\alpha)}\left(L_{t} \geq l\right) & =\mathbb{P}_{x}^{(-\alpha)}\left(\tau_{l} \leq t\right) \\
& =e^{-\alpha l} \frac{R_{-r^{2}}(0,0)}{R_{-r^{2}}(0, x)} \mathbb{E}_{x}\left[e^{r^{2} \tau_{l}} 1_{\left\{\tau_{l} \leq t\right\}}\right] \\
\underset{t \rightarrow+\infty}{\longrightarrow} & \frac{R_{-r^{2}}(0,0)}{R_{-r^{2}}(0, x)} \frac{R_{-r^{2}}(0, x)}{R_{-r^{2}}(0,0)} \exp \left(-l\left(\alpha+\frac{1}{R_{-r^{2}}(0,0)}\right)\right) \\
& =1,
\end{aligned}
$$

using 2.4. As a result, we have $\mathbb{P}_{x}^{(-\alpha)}\left(L_{\infty}=\infty\right)=1$.

§3.4. A few remarks about the penalization by $\left(e^{-\alpha L_{t}}, t \geq 0\right)$

1) To see how the local time at 0 has been reduced, remark that since $X$ is a positively recurrent diffusion on $[0, b], X$ converges in distribution to a random 
variable $X_{\infty}$ whose density is

$$
x \mapsto \frac{m(x)}{m([0, b])} 1_{[0, b]}(x)
$$

(see [BS02, p. 35]). The same is true for $X^{(-\alpha)}: X^{(-\alpha)}$ converges in distribution to a random variable $X_{\infty}^{(-\alpha)}$ whose density is

$$
x \mapsto \frac{\left(\mathbb{E}_{x}\left[e^{r^{2} T_{0}}\right]\right)^{2} m(x)}{m^{(-\alpha)}([0, b])} 1_{[0, b]}(x) .
$$

Then, since $x \mapsto \mathbb{E}_{x}\left[e^{r^{2} T_{0}}\right]$ is an increasing function, we have, for $\varepsilon \leq b$,

$$
\begin{aligned}
\mathbb{P}\left(X_{\infty}^{(-\alpha)}<\varepsilon\right) & =\frac{1}{m^{(-\alpha)}([0, b])} \int_{0}^{\varepsilon}\left(\mathbb{E}_{x}\left[e^{r^{2} T_{0}}\right]\right)^{2} m(x) d x \\
& \leq \frac{1}{m^{(-\alpha)}([0, b])}\left(\mathbb{E}_{\varepsilon}\left[e^{r^{2} T_{0}}\right]\right)^{2} m([0, \varepsilon]) .
\end{aligned}
$$

But, by the first mean integral formula, there is $\delta \in] 0, b[$ such that

$$
\int_{0}^{b}\left(\mathbb{E}_{x}\left[e^{r^{2} T_{0}}\right]\right)^{2} m(x) d x=\left(\mathbb{E}_{\delta}\left[e^{r^{2} T_{0}}\right]\right)^{2} \int_{0}^{b} m(x) d x
$$

This implies

$$
m^{(-\alpha)}([0, b])=\left(\mathbb{E}_{\delta}\left[e^{r^{2} T_{0}}\right]\right)^{2} m([0, b]) .
$$

Therefore, plugging 3.20 in 3.19 , we see that, for $\varepsilon<\delta$,

$$
\begin{aligned}
\mathbb{P}\left(X_{\infty}^{(-\alpha)}<\varepsilon\right) & \leq \frac{\left(\mathbb{E}_{\varepsilon}\left[e^{r^{2} T_{0}}\right]\right)^{2}}{\left(\mathbb{E}_{\delta}\left[e^{r^{2} T_{0}}\right]\right)^{2}} \frac{m([0, \varepsilon])}{m([0, b])} \\
& =\left(\frac{\mathbb{E}_{\varepsilon}\left[e^{r^{2} T_{0}}\right]}{\mathbb{E}_{\delta}\left[e^{r^{2} T_{0}}\right]}\right)^{2} \mathbb{P}\left(X_{\infty}<\varepsilon\right)<\mathbb{P}\left(X_{\infty}<\varepsilon\right) .
\end{aligned}
$$

Heuristically, this means that the penalized diffusion spends less time in the vicinity of 0 than the original one.

2) For this class of diffusions, the penalization by a decreasing exponential function is not sufficient to make the local time at 0 finite. A quite natural idea is to let $r$ tend to $\omega_{1}$ (i.e. $\alpha$ to $+\infty$ ). In this case, for $x \neq 0$, identity 1.4 has to be replaced by

$$
\mathbb{P}_{x}\left(L_{t}=0\right)=\mathbb{P}_{x}\left(T_{0}>t\right) \underset{t \rightarrow+\infty}{\sim}-\frac{1}{\omega_{1}^{2}} \Psi\left(x,-\omega_{1}^{2}\right) \frac{1}{\left.\frac{\partial}{\partial z} R_{z}(0,0)\right|_{z=-\omega_{1}^{2}}} \exp \left(-\omega_{1}^{2} t\right) .
$$

The penalization by $\left(1_{\left\{T_{0}>t\right\}}, t \geq 0\right)$ then yields the martingale

$$
M_{s}^{(-\infty)}=\exp \left(-\omega_{1}^{2} s\right) \frac{\Psi\left(X_{s},-\omega_{1}^{2}\right)}{\Psi\left(x,-\omega_{1}^{2}\right)} 1_{\left\{T_{0}>s\right\}}
$$


and we have actually $\mathbb{P}_{x}^{(-\infty)}\left(L_{\infty}=0\right)=1$. This time, the penalization is too strong. An intermediate case would probably be given by a penalization with $\left(1_{\left\{L_{t}<l\right\}}, t \geq 0\right)$ for $\left.l \in\right] 0,+\infty[$, but we have not been able to settle it yet.

$\S 3.5$. Short proof of the penalization by $\left(e^{\alpha L_{t}}, t \geq 0\right)$

Let us mention first that, formally, the formulae of the penalization with $\left(e^{\alpha L_{t}}\right.$, $t \geq 0)$ can be deduced from the ones with $\left(e^{-\alpha L_{t}}, t \geq 0\right)$ on replacing $-\alpha$ by $\alpha$ and $r$ by $i \rho$. In this case, Assumption 2.1 has to be replaced by

Assumption 3.1. We assume that for every $d>0$, there is $\beta>0$ such that, for $z \in\{z \in \mathbb{C} ; 0 \leq \operatorname{Re}(z) \leq d\}$

$$
R_{z}(0,0) \underset{|z| \rightarrow+\infty}{=} \mathcal{O}\left(\frac{1}{|z|^{\beta}}\right) .
$$

The line of the proof in this case is very close to the one given in the previous sections. However we must take care of integrability problems. First, for $\lambda \in \mathbb{R}_{+}$, $\lambda \mapsto R_{\lambda}(0,0)=\int_{0}^{\infty} e^{-\lambda t} p(t, 0,0) d t$ is a continuous and strictly decreasing function, which tends to $+\infty$ at 0 according to Lemma 2.2. and to 0 at $+\infty$ by the monotone convergence theorem. It is thus a bijection from $\mathbb{R}_{+}^{*}$ to $\mathbb{R}_{+}^{*}$, and the equation $1 / R_{\lambda^{2}}(0,0)=\alpha$ admits a unique positive solution, which we denote by $\rho$.

Next, note that, by Jensen's inequality,

$$
\frac{\left(-\rho^{2} \mathbb{E}_{0}\left[\tau_{l}\right]\right)^{k}}{k !} \leq \frac{\mathbb{E}_{0}\left[\left(-\rho^{2} \tau_{l}\right)^{k}\right]}{k !} \quad(l>0, k \in \mathbb{N}),
$$

and following the same sequence of identities as in $3.9-3.12$ gives

$$
\rho^{2} R_{\rho^{2}}(0,0) m([0, b])>1
$$

since $R_{\rho^{2}}(0,0)=1 / \alpha>0$, and

$$
\lim _{t \rightarrow+\infty}\left(1-\frac{\rho^{2} t}{\alpha L_{t}}\right)=1-\frac{\rho^{2} m([0, b])}{\alpha}=1-\rho^{2} R_{\rho^{2}}(0,0) m([0, b])<0 \quad \mathbb{P} \text {-a.s. }
$$

Note that 3.21 and the fact that $\lambda \mapsto \lambda R_{\rho^{2}}(0,0) m([0, b])$ is an increasing function of $\lambda$ imply that, for $\lambda>\rho^{2}$,

$$
-\lambda t+\alpha L_{t}=\alpha L_{t}\left(1-\frac{\lambda t}{\alpha L_{t}}\right) \underset{t \rightarrow+\infty}{\sim} \alpha L_{t}\left(1-\lambda R_{\rho^{2}}(0,0) m([0, b])\right) \underset{t \rightarrow+\infty}{\longrightarrow}-\infty .
$$

Let $\lambda>\rho^{2}$. Consequently,

$$
\begin{aligned}
\int_{0}^{\infty} e^{-\lambda t} e^{\alpha L_{t}} d t & =-\frac{1}{\lambda}\left[e^{-\lambda t} e^{\alpha L_{t}}\right]_{0}^{+\infty}+\frac{\alpha}{\lambda} \int_{0}^{\infty} e^{-\lambda t} e^{\alpha L_{t}} d L_{t} \\
& =\frac{1}{\lambda}+\frac{\alpha}{\lambda} \int_{0}^{\infty} e^{-\lambda \tau_{l}} e^{\alpha l} d l
\end{aligned}
$$


Integrating this identity with respect to $d \mathbb{P}_{x}$ on $\Omega$, and applying the Fubini-Tonelli theorem, leads to

$$
\begin{aligned}
\int_{0}^{\infty} e^{-\lambda t} \mathbb{E}_{x}\left[e^{\alpha L_{t}}\right] d t & =\frac{1}{\lambda}+\frac{\alpha}{\lambda} \int_{0}^{\infty} \mathbb{E}_{x}\left[e^{-\lambda \tau_{l}}\right] e^{\alpha l} d l \\
& =\frac{1}{\lambda}+\frac{\alpha}{\lambda} \frac{R_{\lambda}(0, x)}{R_{\lambda}(0,0)} \int_{0}^{\infty} e^{\left(-1 / R_{\lambda}(0,0)+\alpha\right) l} d l \quad(<\infty) \\
& =\frac{1}{\lambda}+\frac{R_{\lambda}(0, x)}{\lambda R_{\lambda}(0,0)} \frac{\alpha}{\frac{1}{R_{\lambda}(0,0)}-\alpha}
\end{aligned}
$$

We deduce in particular that for all $t \geq 0, \mathbb{E}_{x}\left[e^{\alpha L_{t}}\right]<\infty$ a.s. Now, to mimic the proof of Theorem 1.1 , we have to overcome the problem that $t \mapsto \mathbb{E}\left[e^{\alpha L_{t}}\right]$ is no longer integrable on $\mathbb{R}_{+}$. We choose a real $d>\rho$, and we study the asymptotics of the function $t \mapsto e^{-d^{2} t} \mathbb{E}\left[e^{\alpha L_{t}}\right]$ (which now belongs to $L^{1}\left(\mathbb{R}_{+}\right)$). This amounts to translating the Laplace transform towards the negative reals:

$$
\int_{0}^{\infty} e^{-\lambda t} e^{-d^{2} t} \mathbb{E}_{x}\left[e^{\alpha L_{t}}\right] d t=\frac{1}{\lambda+d^{2}}+\frac{R_{\lambda+d^{2}}(0, x)}{\left(\lambda+d^{2}\right) R_{\lambda+d^{2}}(0,0)} \frac{\alpha}{\frac{1}{R_{\lambda+d^{2}}(0,0)}-\alpha}
$$

We then apply the residue theorem around the pole $\lambda=-\left(d^{2}-\rho^{2}\right)<0$ and notice that the artificial weight $e^{-d^{2} t}$ cancels in the final equivalent.

\section{$\S 4$. Proof of Theorem 1.4}

Let $\alpha, \beta>0$, and let $r^{2}$ be defined by 1.2 . In this section, we shall only make the proof of the penalization of the measure $\mathbb{P}^{(-\alpha)}$ by $\left(e^{ \pm \beta L_{t}}, t \geq 0\right)$. From Theorem 1.3. under $\mathbb{P}_{x}^{(-\alpha)}$, the coordinate process $\left(X_{t}, t \geq 0\right)$ is still a positively recurrent diffusion reflected on $[0, b]$. We still write $\mathbb{P}_{x}^{(-\alpha)}$ for the equivalent probability under which $\left(X_{t}, t \geq 0\right)$ is on natural scale.

Hence, Theorem 1.2 applies and we can perform the penalization of $\mathbb{P}_{x}^{(-\alpha)}$ by $\left(e^{ \pm \beta L_{t}}, t \geq 0\right)$.

$$
\text { §4.1. Penalization of } \mathbb{P}_{x}^{(-\alpha)} \text { by }\left(e^{-\beta L_{t}}, t \geq 0\right)
$$

Denoting by $M^{(-\alpha)(-\beta)}$ the $\mathbb{P}_{x}^{(-\alpha)}$-martingale given by

$$
\left(M_{t}^{(-\alpha)(-\beta)}:=\exp \left(\sigma^{2} t-\beta L_{t}\right) \frac{R_{-\sigma^{2}}^{(-\alpha)}\left(0, X_{t}\right)}{R_{-\sigma^{2}}^{(-\alpha)}(0, x)}, t \geq 0\right)
$$

where $R^{(-\alpha)}$ is the resolvent kernel of $X$ under $\mathbb{P}^{(-\alpha)}$ and $\sigma^{2}$ is the solution of smallest modulus of the equation 


$$
\beta+\frac{1}{R_{-\sigma^{2}}^{(-\alpha)}(0,0)}=0
$$

there exists a family $\left(\mathbb{P}_{x}^{(-\alpha)(-\beta)}\right)_{x \in[0, b]}$ of probabilities defined on $\left(\Omega, \mathcal{F}_{\infty}\right)$ such that

$$
\mathbb{P}_{x}^{(-\alpha)(-\beta)}\left(\Lambda_{u}\right)=\mathbb{E}_{x}^{(-\alpha)}\left[1_{\Lambda_{u}} M_{u}^{(-\alpha)(-\beta)}\right] \quad \text { for every } u \geq 0 \text { and every } \Lambda_{u} \in \mathcal{F}_{u} .
$$

But, for $\lambda \geq 0$,

$$
\begin{aligned}
\mathbb{E}_{x}^{(-\alpha)}\left[e^{-\lambda T_{0}} 1_{\left\{T_{0} \leq t\right\}}\right] & =\mathbb{E}_{x}\left[e^{-\lambda T_{0}} 1_{\left\{T_{0} \leq t\right\}} M_{t}^{(-\alpha)}\right]=\mathbb{E}_{x}\left[e^{-\lambda T_{0}} 1_{\left\{T_{0} \leq t\right\}} M_{T_{0}}^{(-\alpha)}\right] \\
& =\frac{R_{-r^{2}}(0,0)}{R_{-r^{2}}(0, x)} \mathbb{E}_{x}\left[e^{-\left(\lambda-r^{2}\right) T_{0}} 1_{\left\{T_{0} \leq t\right\}}\right]
\end{aligned}
$$

from Doob's optional stopping theorem. Then, letting $t$ tend to $+\infty$ on both sides, and applying the monotone convergence theorem, we obtain, from 2.3,

$$
\frac{R_{\lambda}^{(-\alpha)}(0, x)}{R_{\lambda}^{(-\alpha)}(0,0)}=\frac{R_{-r^{2}}(0,0)}{R_{-r^{2}}(0, x)} \frac{R_{\lambda-r^{2}}(0, x)}{R_{\lambda-r^{2}}(0,0)} .
$$

Therefore,

$$
M_{t}^{(-\alpha)(-\beta)}=\exp \left(\sigma^{2} t-\beta L_{t}\right) \frac{R_{-r^{2}}(0, x)}{R_{-\left(\sigma^{2}+r^{2}\right)}(0, x)} \frac{R_{-\left(\sigma^{2}+r^{2}\right)}\left(0, X_{t}\right)}{R_{-r^{2}}\left(0, X_{t}\right)} \quad(t \geq 0),
$$

and, for $\Lambda_{s} \in \mathcal{F}_{s}$, we have

$$
\begin{aligned}
\mathbb{P}_{x}^{(-\alpha)(-\beta)}\left(\Lambda_{s}\right) & =\mathbb{E}_{x}^{(-\alpha)}\left[1_{\Lambda_{s}} \exp \left(\sigma^{2} s-\beta L_{s}\right) \frac{R_{-r^{2}}(0, x)}{R_{-\left(\sigma^{2}+r^{2}\right)}(0, x)} \frac{R_{-\left(\sigma^{2}+r^{2}\right)}\left(0, X_{s}\right)}{R_{-r^{2}}\left(0, X_{s}\right)}\right] \\
& =\mathbb{E}_{x}\left[1_{\Lambda_{s}} \exp \left(\left(\sigma^{2}+r^{2}\right) s+(-\beta-\alpha) L_{s}\right) \frac{R_{-\left(\sigma^{2}+r^{2}\right)}\left(0, X_{s}\right)}{R_{-\left(\sigma^{2}+r^{2}\right)}(0, x)}\right] .
\end{aligned}
$$

Now, the comparison of 2.3 and 3.18 gives

$$
\mathbb{E}_{0}^{(-\alpha)}\left[e^{-\lambda \tau_{l}}\right]=\exp \left(-l / R_{\lambda}^{(-\alpha)}(0,0)\right)=\exp \left(-l\left(\alpha+\frac{1}{R_{\lambda-r^{2}}(0,0)}\right)\right),
$$

which yields

$$
\frac{1}{R_{\lambda}^{(-\alpha)}(0,0)}=\alpha+\frac{1}{R_{\lambda-r^{2}}(0,0)} .
$$

Therefore, setting $\xi^{2}:=\sigma^{2}+r^{2}$, the equation 4.1 satisfied by $\sigma^{2}$ can be rewritten as

$$
\beta+\alpha+\frac{1}{R_{-\xi^{2}}(0,0)}=0
$$


and $\xi^{2}$ is the smallest solution of 4.5 . Indeed, otherwise, there would exist $u^{2}$ such that $u^{2}<\sigma^{2}+r^{2}$ and $\beta+\alpha+1 / R_{-u^{2}}(0,0)=0$. But, from (4.4), this would imply that $\beta+1 / R_{-\left(u^{2}-r^{2}\right)}^{(-\alpha)}(0,0)=0$, which contradicts the fact that $\sigma^{2}$ is the smallest solution of this equation (i.e. (4.1)). (Note that $u^{2}-r^{2}$ must be positive, since $\lambda \mapsto R_{\lambda}(0,0)$ takes positive values on $[0,+\infty[)$. Finally, from 4.3,

$$
\begin{aligned}
\mathbb{P}_{x}^{(-\alpha)(-\beta)}\left(\Lambda_{s}\right) & =\mathbb{E}_{x}\left[1_{\Lambda_{s}} \exp \left(\xi^{2} s+(-\beta-\alpha) L_{s}\right) \frac{R_{-\xi^{2}}\left(0, X_{s}\right)}{R_{-\xi^{2}}(0, x)}\right] \\
& =\mathbb{E}_{x}\left[1_{\Lambda_{s}} M_{s}^{(-\alpha-\beta)}\right]=\mathbb{P}_{x}^{(-\alpha-\beta)}\left(\Lambda_{s}\right) .
\end{aligned}
$$

$\S$ 4.2. Penalization of $\mathbb{P}_{x}^{(-\alpha)}$ by $\left(e^{\beta L_{t}}, t \geq 0\right)$

Now, if we penalize $\mathbb{P}_{x}^{(-\alpha)}$ by $\left(e^{\beta L_{t}}, t \geq 0\right)$, we obtain the family $\left(\mathbb{P}_{x}^{(-\alpha)(\beta)}\right)_{x \in[0, b]}$ of probabilities defined on $\left(\Omega, \mathcal{F}_{\infty}\right)$ by

$$
\mathbb{P}_{x}^{(-\alpha)(\beta)}\left(\Lambda_{u}\right)=\mathbb{E}_{x}^{(-\alpha)}\left[1_{\Lambda_{u}} M_{u}^{(-\alpha)(\beta)}\right] \quad \text { for every } u \geq 0 \text { and every } \Lambda_{u} \in \mathcal{F}_{u},
$$

where $M^{(-\alpha)(\beta)}$ is the $\mathbb{P}_{x}^{(-\alpha)}$-martingale given by

$$
M_{t}^{(-\alpha)(\beta)}:=\exp \left(-\eta^{2} t+\beta L_{t}\right) \frac{R_{\eta^{2}}^{(-\alpha)}\left(0, X_{t}\right)}{R_{\eta^{2}}^{(-\alpha)}(0, x)} \quad(t \geq 0)
$$

with $\eta^{2}$ the unique solution of the equation $1 / R_{\eta^{2}}^{(-\alpha)}(0,0)=\beta$. From 4.2 , $M^{(-\alpha)(\beta)}$ can be rewritten as

$$
M_{t}^{(-\alpha)(\beta)}=\exp \left(-\eta^{2} t+\beta L_{t}\right) \frac{R_{-r^{2}}(0, x)}{R_{\eta^{2}-r^{2}}(0, x)} \frac{R_{\eta^{2}-r^{2}}\left(0, X_{t}\right)}{R_{-r^{2}}\left(0, X_{t}\right)} \quad(t \geq 0)
$$

and, for $\Lambda_{s} \in \mathcal{F}_{s}$, we have

$$
\begin{aligned}
\mathbb{P}_{x}^{(-\alpha)(\beta)}\left(\Lambda_{s}\right) & =\mathbb{E}_{x}^{(-\alpha)}\left[1_{\Lambda_{s}} \exp \left(-\eta^{2} s+\beta L_{s}\right) \frac{R_{-r^{2}}(0, x)}{R_{\eta^{2}-r^{2}}(0, x)} \frac{R_{\eta^{2}-r^{2}}\left(0, X_{s}\right)}{R_{-r^{2}}\left(0, X_{s}\right)}\right] \\
& =\mathbb{E}_{x}\left[1_{\Lambda_{s}} \exp \left(\left(\eta^{2}-r^{2}\right) s+(\beta-\alpha) L_{s}\right) \frac{R_{\eta^{2}-r^{2}}\left(0, X_{s}\right)}{R_{\eta^{2}-r^{2}}(0, x)}\right] .
\end{aligned}
$$

From $4.5, \eta^{2}-r^{2}$ is a solution of the equation

$$
\alpha-\beta+\frac{1}{R_{\eta^{2}-r^{2}}(0,0)}=0 .
$$

Thus, if $\beta \geq \alpha$, then $\eta^{2}-r^{2}=\zeta^{2} \geq 0$ is the unique solution of $\alpha-\beta+1 / R_{\zeta^{2}}(0,0)$ $=0$, and

$$
\mathbb{P}_{x}^{(-\alpha)(\beta)}\left(\Lambda_{s}\right)=\mathbb{E}_{x}\left[1_{\Lambda_{s}} \exp \left(\zeta^{2} s+(\beta-\alpha) L_{s}\right) \frac{R_{\zeta^{2}}\left(0, X_{s}\right)}{R_{\zeta^{2}}(0, x)}\right]=\mathbb{P}_{x}^{(\beta-\alpha)}\left(\Lambda_{s}\right) .
$$


On the other hand, if $\beta \leq \alpha$, the same proof shows that $\eta^{2}-r^{2}=-\zeta^{2} \leq 0$ is the smallest solution of $\alpha-\beta+1 / R_{-\zeta^{2}}(0,0)=0$ and

$$
\mathbb{P}_{x}^{(-\alpha)(\beta)}\left(\Lambda_{s}\right)=\mathbb{E}_{x}\left[1_{\Lambda_{s}} \exp \left(-\zeta^{2} s+(\beta-\alpha) L_{s}\right) \frac{R_{-\zeta^{2}}\left(0, X_{s}\right)}{R_{-\zeta^{2}}(0, x)}\right]=\mathbb{P}_{x}^{(\beta-\alpha)}\left(\Lambda_{s}\right)
$$

The other cases can be dealt with in the same way.

\section{$\S 5$. Application to Bessel processes of dimension $\delta \in] 0,2[$ reflected at 1}

\section{$\S 5.1$. The general case}

Let $Y^{(\nu)}$ be a Bessel process of index $\left.\nu=\delta / 2-1 \in\right]-1,0[$ reflected at 1 . Then $Y^{(\nu)}$ is a positively recurrent diffusion, with infinitesimal generator

$$
\mathcal{G}_{Y}^{(\nu)}=\frac{1}{2} \frac{\partial^{2}}{\partial y^{2}}+\frac{2 \nu+1}{2 y} \frac{\partial}{\partial y} .
$$

Its speed measure and scale function are given by

$$
m_{Y}(d y)=\frac{y^{2 \nu+1}}{|\nu|} d y \quad \text { and } \quad s_{Y}(y)=y^{-2 \nu}
$$

We define $\left(X_{t}:=s\left(Y_{t}^{(\nu)}\right), t \geq 0\right)$. Then $X$ is a diffusion on natural scale. Its infinitesimal generator $\mathcal{G}$ is given, for $f$ a bounded function defined on $\mathbb{R}_{+}$and of class $\mathcal{C}^{2}$, by

$$
\mathcal{G} f(x)=2 \nu^{2} x^{2+1 / \nu} f^{\prime \prime}(x) .
$$

Thus, its speed measure equals $m(d x)=\left(2 \nu^{2}\right)^{-1} x^{-2-1 / \nu} d x$. We now determine the two eigenfunctions $\Phi$ and $\Psi$ solving $(2.6)$. Let us introduce

$$
\left.I_{\nu}(z):=\sum_{k=0}^{\infty} \frac{(z / 2)^{\nu+2 k}}{\Gamma(k+1) \Gamma(k+\nu+1)}, \quad z \in \mathbb{C} \backslash\right]-\infty, 0[,
$$

the modified Bessel function of the first kind, and

$$
\left.K_{\nu}(z):=\frac{\pi}{2} \frac{I_{-\nu}(z)-I_{\nu}(z)}{\sin (\nu \pi)}, \quad z \in \mathbb{C} \backslash\right]-\infty, 0[, \nu \notin \mathbb{Z}
$$

the MacDonald function. It is known (see N. N. Lebedev Leb72, Chapter 5.7, p. 110]) that these two functions generate the set of solutions of the linear differential equation

$$
u^{\prime \prime}+\frac{1}{x} u^{\prime}-\left(1+\frac{\nu^{2}}{x^{2}}\right) u=0 .
$$

It is then not too difficult to verify that

$$
x \mapsto \sqrt{x} I_{\nu}\left(\sqrt{2 \lambda} x^{-1 / 2 \nu}\right) \quad \text { and } \quad x \mapsto \sqrt{x} K_{\nu}\left(\sqrt{2 \lambda} x^{-1 / 2 \nu}\right)
$$


generate the set of eigenfunctions of $\mathcal{G}$ associated with the eigenvalue $\lambda$. The boundary conditions 2.6 yield next

$$
\Phi(x, \lambda)=\left(\frac{2}{\sqrt{2 \lambda}}\right)^{\nu} \Gamma(1+\nu) \sqrt{x} I_{\nu}\left(\sqrt{2 \lambda} x^{-1 / 2 \nu}\right)
$$

and

$$
\begin{aligned}
\Psi(x, \lambda)= & \left(\frac{\sqrt{2 \lambda}}{2}\right)^{\nu} \Gamma(1-\nu) \sqrt{x} I_{\nu}\left(\sqrt{2 \lambda} x^{-1 / 2 \nu}\right) \\
& +\frac{2 \nu}{\Gamma(1+\nu)}\left(\frac{\sqrt{2 \lambda}}{2}\right)^{\nu} \sqrt{x} K_{\nu}\left(\sqrt{2 \lambda} x^{-1 / 2 \nu}\right) .
\end{aligned}
$$

Hence, we deduce from (2.9) with $b=1$ that

$$
\begin{aligned}
R_{\lambda}(0,0) & :=\frac{\Psi^{\prime}(1, \lambda)}{\Phi^{\prime}(1, \lambda)} \\
& =\frac{-\nu}{\Gamma(1+\nu)}\left(\frac{\sqrt{2 \lambda}}{2}\right)^{2 \nu}\left(\Gamma(-\nu)+\frac{2}{\Gamma(1+\nu)} \frac{K_{\nu+1}(\sqrt{2 \lambda})}{I_{\nu+1}(\sqrt{2 \lambda})}\right) .
\end{aligned}
$$

We also introduce, for $\nu \in]-1,0[$, the Bessel function of the first kind, which is defined on $\mathbb{C}$ by

$$
J_{\nu}(z):=\sum_{k=0}^{\infty} \frac{(-1)^{k}(z / 2)^{\nu+2 k}}{\Gamma(k+1) \Gamma(k+\nu+1)} .
$$

Then, for $z \in \mathbb{C}$ such that $-\pi / 2<\arg (z)<\pi$, we have

$$
J_{\nu}(z):=e^{-\nu \pi i / 2} I_{\nu}(i z)
$$

(see N. N. Lebedev Leb72, pp. 109 and 113]).

With this notation, we can now state the following version of Theorem 1.1

Theorem 5.1. Let $Y^{(\nu)}$ be a Bessel process of index $\left.\nu \in\right]-1,0[$ reflected at 1 , $\left(X:=\left(Y_{t}^{(\nu)}\right)^{-2 \nu}, t \geq 0\right)$ and $\alpha>0$.

(i) Let $r^{2}$ be the solution of smallest modulus of the equation $\alpha+1 / R_{-r^{2}}(0,0)=0$. Then

$$
\mathbb{E}_{x}\left[e^{-\alpha L_{t}(X)}\right] \underset{t \rightarrow+\infty}{\sim} \exp \left(-r^{2} t\right)\left(\frac{\Phi\left(x,-r^{2}\right)}{\alpha}+\Psi\left(x,-r^{2}\right)\right) c_{-}(\alpha, \nu, r)
$$

where

$$
c_{-}(\alpha, \nu, r)=\frac{1}{-\frac{\nu}{\alpha}-\frac{\nu}{(\Gamma(1+\nu))^{2}}\left(\frac{r^{2}}{2}\right)^{\nu} \frac{1}{J_{\nu+1}^{2}(r \sqrt{2})}} .
$$


(ii) Let $\rho^{2}$ be the unique solution in $\mathbb{R}_{+}$of $-\alpha+1 / R_{\rho^{2}}(0,0)=0$. Then

$$
\mathbb{E}_{x}\left[e^{\alpha L_{t}(X)}\right] \underset{t \rightarrow+\infty}{\sim} \exp \left(\rho^{2} t\right)\left(\frac{\Phi\left(x, \rho^{2}\right)}{\alpha}-\Psi\left(x, \rho^{2}\right)\right) c_{+}(\alpha, \nu, r)
$$

where

$$
c_{+}(\alpha, \nu, r)=\frac{1}{-\frac{\nu}{\alpha}-\frac{\nu}{(\Gamma(1+\nu))^{2}}\left(\frac{\rho^{2}}{2}\right)^{\nu} \frac{1}{I_{\nu+1}^{2}(\rho \sqrt{2})}} .
$$

Note that to simplify the presentation, we used the identity $2.8, R_{\lambda}(x, y)=$ $\Phi(x, \lambda)\left(R_{\lambda}(0,0) \Phi(y, \lambda)-\Psi(y, \lambda)\right)$, in the above formulas. Likewise, the computation of $\frac{\partial}{\partial z} R_{z}(0,0)$ can be significantly illuminated by using the following identity for the Wronskian of $I_{\nu}$ and $K_{\nu}$ :

$$
W\left(I_{\nu}(z), K_{\nu}(z)\right):=K_{\nu}^{\prime}(z) I_{\nu}(z)-I_{\nu}^{\prime}(z) K_{\nu}(z)=-1 / z
$$

Proof of Theorem 5.1. We only need to check that Assumptions 2.1 and 3.1 are satisfied in this set-up, in order to apply Theorem 1.1 .

Let us denote by $\left(\omega_{n}\right)_{n \geq 1}$ the zeros of $R_{-\lambda^{2}}(0,0)$, and let $c, d \in \mathbb{R}$ be such that $[c, d] \subset]-\omega_{1}^{2},+\infty[$, and $z \in\{z \in \mathbb{C} ; z=a+i v, c \leq a \leq d\}$. We are looking for $u \in \mathbb{C}$ such that $u^{2}=2 z=2(a+i v)$. In trigonometrical form, $u$ can be written as

$$
u=\sqrt{2}\left(a^{2}+v^{2}\right)^{1 / 4} \exp \left(\frac{i}{2} \arg (2(a+i v))\right) .
$$

Now, since $|z|=\sqrt{a^{2}+v^{2}}$ and $a$ is bounded in $[c, d],|z| \rightarrow+\infty$ implies that $v \rightarrow \pm \infty$.

First, we assume that $v$ tends to $+\infty$. Then $\arg (2(a+i v)) \underset{v \rightarrow+\infty}{\longrightarrow} \pi / 2$, so from (5.4) we obtain

$$
u \underset{v \rightarrow+\infty}{\sim} \sqrt{v}+i \sqrt{v}
$$

Therefore, we have

$$
\begin{aligned}
R_{z}(0,0) & =\frac{-\nu}{\Gamma(1+\nu)}\left(\frac{u}{2}\right)^{2 \nu}\left(\Gamma(-\nu)+\frac{2}{\Gamma(1+\nu)} \frac{K_{\nu+1}(u)}{I_{\nu+1}(u)}\right) \\
& \sim \underset{v \rightarrow+\infty}{\sim} \frac{-\nu \Gamma(-\nu)}{\Gamma(1+\nu)}\left(\frac{\sqrt{v}+i \sqrt{v}}{2}\right)^{2 \nu}=\mathcal{O}\left(v^{\nu}\right)
\end{aligned}
$$

since $K_{\nu+1}(u) / I_{\nu+1}(u) \underset{|u| \rightarrow+\infty}{\longrightarrow} 0$ when $|\arg (u)|<\pi / 2-\varepsilon$, according to Leb72, p. 123].

Second, when $v \rightarrow-\infty$, we can prove similarly that $R_{z}(0,0)=\mathcal{O}\left(|v|^{\nu}\right)$. Therefore Assumptions 2.1 and 3.1 hold. 
Of course, the above proof shows that the penalization Theorems 1.2 and 1.3 also hold for Bessel processes of index $\nu \in]-1,0[$ reflected at 1 . We shall not state them once again since all the terms in this framework have already been computed. Instead, we will particularize this set-up to consider the fundamental example of the Brownian motion reflected on $[0,1]$.

\section{§5.2. Brownian motion reflected on $[0,1]$}

The resolvent kernel (5.3) and the eigenfunctions (5.1) and (5.2) of the infinitesimal generator $\mathcal{G}$ reduce significantly when $\nu=-1 / 2$ (i.e. the Brownian motion case). Indeed, as

$$
I_{-1 / 2}(z)=\sqrt{\frac{2}{\pi z}} \cosh (z), \quad I_{1 / 2}(z)=\sqrt{\frac{2}{\pi z}} \sinh (z) \quad \text { and } \quad K_{-1 / 2}(z)=\sqrt{\frac{\pi}{2 z}} e^{-z}
$$

we get, by substituting in $5.1-5.3$,

$$
\Phi(x, \lambda)=\cosh (\sqrt{2 \lambda} x), \quad \Psi(x, \lambda)=\frac{1}{\sqrt{2 \lambda}} \sinh (\sqrt{2 \lambda} x),
$$

and

$$
R_{\lambda}(0,0)=\frac{1}{\sqrt{2 \lambda} \tanh (\sqrt{2 \lambda})}=\frac{\sum_{n \geq 0}(2 \lambda)^{n} /(2 n) !}{\sum_{n \geq 0}(2 \lambda)^{n+1} /(2 n+1) !} .
$$

In this particular setting, we have:

Theorem 5.2. Let $X$ be a Brownian motion reflected on $[0,1]$ and $\alpha>0$.

(i) Let $r$ be the unique solution in $] 0, \pi /(2 \sqrt{2})[$ of the equation $\alpha=\sqrt{2} r \tan (\sqrt{2} r)$. Then

$$
\mathbb{E}_{x}\left[e^{-\alpha L_{t}}\right] \underset{t \rightarrow+\infty}{\sim} \exp \left(-r^{2} t\right) \frac{\cos (\sqrt{2} r(1-x))}{\cos (\sqrt{2} r)} \frac{2 \alpha}{2 r^{2}+\alpha+\alpha^{2}} .
$$

(ii) Let $\rho$ be the unique solution in $] 0,+\infty[$ of the equation $\alpha=\sqrt{2} \rho \tanh (\sqrt{2} \rho)$. Then

$$
\mathbb{E}_{x}\left[e^{\alpha L_{t}}\right] \underset{t \rightarrow+\infty}{\sim} \exp \left(\rho^{2} t\right) \frac{\cosh (\sqrt{2} \rho(1-x))}{\cosh (\sqrt{2} \rho)} \frac{2 \alpha}{2 \rho^{2}+\alpha-\alpha^{2}} .
$$

Theorem 5.3. Let $X$ be a Brownian motion reflected on $[0,1]$ and $\alpha>0$.

(i) The processes

$$
\left(M_{t}^{(-\alpha)}:=\exp \left(r^{2} t-\alpha L_{t}\right) \frac{\cos \left(\sqrt{2} r\left(1-X_{t}\right)\right)}{\cos (\sqrt{2} r(1-x))}, t \geq 0\right)
$$

and

$$
\left(M_{t}^{(\alpha)}:=\exp \left(-\rho^{2} t+\alpha L_{t}\right) \frac{\cosh \left(\sqrt{2} \rho\left(1-X_{t}\right)\right)}{\cosh (\sqrt{2} \rho(1-x))}, t \geq 0\right)
$$

are continuous, strictly positive $\mathbb{P}_{x}$-martingales which converge to 0 as $t \rightarrow+\infty$. 
(ii) Let $s>0$ and $x \in[0,1]$. For every $\Lambda_{s} \in \mathcal{F}_{s}$, we have

$$
\lim _{t \rightarrow+\infty} \frac{\mathbb{E}_{x}\left[1_{\Lambda_{s}} e^{ \pm \alpha L_{t}}\right]}{\mathbb{E}_{x}\left[e^{ \pm \alpha L_{t}}\right]}=\mathbb{E}_{x}\left[1_{\Lambda_{s}} M_{s}^{( \pm \alpha)}\right]
$$

(iii) Let $\left(\mathbb{P}_{x}^{( \pm \alpha)}\right)_{x \in[0,1]}$ be the family of probabilities defined on $\left(\Omega, \mathcal{F}_{\infty}\right)$ by

$$
\mathbb{P}_{x}^{( \pm \alpha)}\left(\Lambda_{u}\right)=\mathbb{E}_{x}\left[1_{\Lambda_{u}} M_{u}^{( \pm \alpha)}\right] \quad \text { for every } u \geq 0 \text { and every } \Lambda_{u} \in \mathcal{F}_{u}
$$

Then, under $\mathbb{P}_{x}^{( \pm \alpha)}$, the coordinate process $X$ is a solution of the stochastic differential equation

$$
X_{t}=x+\widetilde{B}_{t}+L_{t}^{0}(X)-L_{t}^{1}(X)+\int_{0}^{t} b^{( \pm \alpha)}\left(X_{s}\right) d s
$$

where $\widetilde{B}$ is a $\mathbb{P}_{x}^{( \pm \alpha)}$-Brownian motion started from 0 and

$$
\left\{\begin{array}{l}
b^{(-\alpha)}(x)=\sqrt{2} r \tan (\sqrt{2} r(1-x)), \\
b^{(+\alpha)}(x)=-\sqrt{2} \rho \tanh (\sqrt{2} \rho(1-x)) .
\end{array}\right.
$$

(iv) Under $\mathbb{P}^{( \pm \alpha)}$, the density of the Lévy measure of the subordinator $\tau$ is given by

$$
\left\{\begin{array}{l}
n^{(-\alpha)}(u)=2 \sum_{n \geq 1} a_{n}^{2} e^{-\left(a_{n}^{2}-r^{2}\right) u} \\
n^{(+\alpha)}(u)=2 \sum_{n \geq 1} a_{n}^{2} e^{-\left(a_{n}^{2}+\rho^{2}\right) u}
\end{array}\right.
$$

where $a_{n}:=\frac{\pi}{2 \sqrt{2}}(2 n-1)$.

Proof. Item (iii) is a direct consequence of (1.6) and merely relies on an application of Girsanov's theorem. Next, to prove (iv), we need to determine the Lévy measure of $\tau$ under $\mathbb{P}$. We use the expansion

$$
\sqrt{2 \lambda} \tanh (\sqrt{2 \lambda})=\sum_{n \geq 1} \frac{2 \lambda}{a_{n}^{2}+\lambda} \quad \text { where } \quad a_{n}=\frac{\pi}{2 \sqrt{2}}(2 n-1)
$$

(see for example H. Cartan Car61, p. 155]). We then write, from 2.3),

$$
\begin{aligned}
\mathbb{E}_{0}\left[e^{-\lambda \tau_{l}}\right] & =\exp \left(-l / R_{\lambda(0,0)}\right)=\exp (-l \sqrt{2 \lambda} \tanh (\sqrt{2 \lambda})) \\
& =\exp \left(-2 l \sum_{n \geq 1} \frac{\lambda}{a_{n}^{2}+\lambda}\right)=\exp \left(-2 l \sum_{n \geq 1} a_{n}^{2}\left(\frac{1}{a_{n}^{2}}-\frac{1}{a_{n}^{2}+\lambda}\right)\right) \\
& =\exp \left(-2 l \sum_{n \geq 1} a_{n}^{2} \int_{0}^{\infty}\left(e^{-a_{n}^{2} u}-e^{-\left(a_{n}^{2}+\lambda\right) u}\right) d u\right) \\
& =\exp \left(-2 l \int_{0}^{\infty}\left(1-e^{-\lambda u}\right) \sum_{n \geq 1} a_{n}^{2} e^{-a_{n}^{2} u} d u\right) .
\end{aligned}
$$


Hence, the density of the Lévy measure of $\tau$ is given by

$$
n(u)=2 \sum_{n \geq 1} a_{n}^{2} e^{-a_{n}^{2} u},
$$

and (iv) is a direct consequence of Theorem 1.3 (ii).

Remark 4. Let us mention that when $X$ is a reflected Brownian motion on $[0,1]$, many equalities in law are known for the subordinator $\tau$. For example, from F. B. Knight [Kni78, Lemma 2.1, p. 436], we have

$$
\begin{aligned}
\tau_{l}(X) & \stackrel{(d)}{=} \int_{0}^{\tau_{l}(|B|)} 1_{00,1[}\left(\left|B_{t}\right|\right) d t \\
& \stackrel{(d)}{=} 2 \int_{0}^{1} L_{\tau_{l}(|B|)}^{a}(|B|) d a \quad \text { (by the occupation time formula) } \\
& \stackrel{(d)}{=} 2 \int_{0}^{1} Z_{t} d t \quad \text { (by the Ray-Knight Theorem), }
\end{aligned}
$$

where $B$ is a standard Brownian motion and $Z$ a squared Bessel process of dimension 0 started from $l$. Moreover, according to P. Carmona, F. Petit and M. Yor CPY01, we have the equality in law

$$
\left(\gamma_{\frac{\pi^{2}}{4} \tau_{l}}, l \geq 0\right) \stackrel{(d)}{=}\left(\xi_{\frac{\pi}{2} l}, l \geq 0\right),
$$

where $\gamma$ is a Brownian motion independent of $\tau$, and $\xi$ is the Lévy process associated by Lamperti's relation with the absolute value of a Cauchy process, whose generator is

$$
L^{\xi} f(\xi)=\frac{1}{\pi} \int_{\mathbb{R}} \frac{\cosh (\eta)}{(\sinh (\eta))^{2}}\left(f(\xi+\eta)-f(\xi)-\eta f^{\prime}(\xi) 1_{\{|\eta| \leq 1\}}\right) d \eta .
$$

In fact, a better knowledge of the law of $\tau_{l}$ (in particular the asymptotic behavior of its distribution) would enable us to penalize the Brownian motion reflected on $[0,1]$ with $\left(1_{\left\{L_{t} \leq l\right\}}, t \geq 0\right)$.

\section{$\S 6$. Other cases}

We have so far studied the penalization of a positively recurrent diffusion reflected on $[0, b]$ by an exponential function of its local time. We shall now briefly deal with null recurrent diffusions and transient diffusions. As previously, the following study will mainly rely on the expressions of the resolvent kernel, as given by Krein's theory. See for example [DM76, Chapter V, p. 162] for an introduction to the Green function, and its expressions in the different situations we shall deal 
with, or KK74 for the original point of view of M. G. Krein and I. S. Kac. But, before starting our discussion related to $b$ and $m([0, b[)$, we mention a Tauberian theorem for Laplace transforms, which we will use several times (see W. Feller [Fel71, Chapter XIII.5, p. 446]):

Theorem 6.1. Let $p \in] 0,+\infty[$. If $f$ is a monotone function on an interval of the form $] x_{0},+\infty[$, then we have the equivalence

$$
\int_{0}^{\infty} e^{-\lambda x} f(x) d x \underset{\lambda \rightarrow 0}{\sim} \frac{1}{\lambda^{p}} \eta\left(\frac{1}{\lambda}\right) \Leftrightarrow f(x) \underset{x \rightarrow+\infty}{\sim} \frac{1}{\Gamma(p)} x^{p-1} \eta(x),
$$

where $\eta$ is a slowly varying function $(i . e$. for all $x>0, \eta(t x) / \eta(t) \rightarrow 1$ as $t \rightarrow+\infty)$.

We shall give below, in each case, an equivalent at 0 of (2.1), and then apply the Tauberian theorem to get an equivalent of $t \mapsto \mathbb{E}_{x}\left[e^{-\alpha L_{t}}\right]$ at $+\infty$. Note that this was not possible for a positively recurrent diffusion reflected on $[0, b]$, as mentioned in Remark 2 .

\section{§6.1. First case: $b=+\infty$ and $m([0,+\infty[)=+\infty$}

Theorem 6.2. Let $X$ be a linear diffusion on natural scale, defined on $[0,+\infty[$ and such that $m([0, x]) \underset{x \rightarrow+\infty}{\sim} x^{1 / \beta-1} \kappa(x)$ with $\left.\beta \in\right] 0,1[$ and $\kappa$ a slowly varying function. Then

$$
\mathbb{E}_{x}\left[e^{-\alpha L_{t}}\right] \underset{t \rightarrow+\infty}{\sim}\left(x+\frac{1}{\alpha}\right) \frac{\eta(t)}{t^{\beta}}
$$

where $\eta$ is another slowly varying function.

Proof. The resolvent kernel takes the form

$$
R_{\lambda}(0,0)=\int_{0}^{\infty} \frac{d x}{\Phi^{2}(x, \lambda)} \underset{\lambda \rightarrow 0}{\longrightarrow}+\infty \quad \text { (using }[2.7)
$$

This implies that $X$ is null recurrent (since $m([0,+\infty[)=+\infty)$. We have

$$
\begin{aligned}
\frac{R_{\lambda}(0, x)}{R_{\lambda}(0,0)} & =\Phi(x, \lambda)-\frac{\Psi(x, \lambda)}{R_{\lambda}(0,0)} \\
& =1+\lambda \int_{0}^{x}(x-s) \Phi(s, \lambda) m(d s)-\frac{x+\lambda \int_{0}^{x}(x-s) \Psi(s, \lambda) m(d s)}{R_{\lambda}(0,0)} .
\end{aligned}
$$

Since $\lim _{\lambda \rightarrow 0} \lambda R_{\lambda}(0,0)=1 / m([0,+\infty[)=0$ (see [BS02, p. 20]), it follows that

$$
\frac{R_{\lambda}(0, x)}{R_{\lambda}(0,0)}=1-\frac{x}{R_{\lambda}(0,0)}+o\left(\frac{1}{R_{\lambda}(0,0)}\right) .
$$


Therefore, plugging 6.3 in 2.1 , we obtain the equivalent:

$$
\begin{aligned}
& \int_{0}^{\infty} e^{-\lambda t} \mathbb{E}_{x}\left[e^{-\alpha L_{t}}\right] d t \\
&= \frac{1}{\lambda}\left[1-\left(1-\frac{x}{R_{\lambda}(0,0)}+o\left(\frac{1}{R_{\lambda}(0,0)}\right)\right)\left(1-\frac{1}{\alpha R_{\lambda}(0,0)}+o\left(\frac{1}{R_{\lambda}(0,0)}\right)\right)\right] \\
& \underset{\lambda \rightarrow 0}{\sim} \frac{x+1 / \alpha}{\lambda R_{\lambda}(0,0)} .
\end{aligned}
$$

Let us now introduce the Lévy measure $\nu$ of the subordinator $\tau$. The measure $\nu$ is absolutely continuous with respect to the Lebesgue measure, with density $n$ which is the Laplace transform of the Borel measure $\sigma$ associated to $m^{-1}$ (the left continuous inverse of $m$ ) by the Krein correspondence:

$$
n(u)=\int_{0}^{\infty} e^{-\xi u} \xi d \sigma(\xi)
$$

(see S. Kotani and S. Watanabe KW82 and F. B. Knight Kni81]). Then the following identity holds:

$$
\frac{1}{R_{\lambda}(0,0)}=\int_{0}^{\infty}\left(1-e^{-\lambda u}\right) n(u) d u
$$

Let $a>0$. We write

$$
\begin{aligned}
\int_{a}^{\infty}\left(1-e^{-\lambda u}\right) n(u) d u & =\left[( e ^ { - \lambda u } - 1 ) \nu \left([u,+\infty[)]_{a}^{+\infty}+\int_{a}^{\infty} \lambda e^{-\lambda u} \nu([u,+\infty[) d u\right.\right. \\
& =\left(1-e^{-\lambda a}\right) \nu\left(\left[a,+\infty[)+\int_{a}^{\infty} \lambda e^{-\lambda u} \nu([u,+\infty[) d u .\right.\right.
\end{aligned}
$$

The two terms being positive, we can deduce, letting $a \rightarrow 0$,

$$
\frac{1}{\lambda R_{\lambda}(0,0)}=\int_{0}^{\infty} e^{-\lambda u} \nu([u,+\infty[) d u+c,
$$

where $c:=\lim _{a \rightarrow 0} a \nu([a,+\infty[)<\infty$. Observe that $c=0$. Indeed, otherwise, if $c>0$, we would have $\nu([a,+\infty[) \underset{a \rightarrow 0}{\sim} c / a$ and

$$
\begin{aligned}
\int_{a}^{1} u \nu(d u) & =[-u \nu([u, 1])]_{a}^{1}+\int_{a}^{1} \nu([u, 1]) d u \\
& =a \nu([a, 1])+\int_{a}^{1} \nu([u, 1]) d u \underset{a \rightarrow 0}{\longrightarrow}+\infty
\end{aligned}
$$

since $a \mapsto \nu([a, 1])$ would not be integrable at 0 . But this contradicts the fact that $\nu$ is the Lévy measure of a subordinator, i.e. $\int_{0}^{+\infty}(u \wedge 1) \nu(d u)<\infty$. Therefore, 
from $(6.4)$ and 6.7 we obtain

$$
\int_{0}^{\infty} e^{-\lambda t} \mathbb{E}_{x}\left[e^{-\alpha L_{t}}\right] d t \underset{\lambda \rightarrow 0}{\sim}\left(x+\frac{1}{\alpha}\right) \int_{0}^{\infty} e^{-\lambda u} \nu([u,+\infty[) d u,
$$

and it remains to find an equivalent of the RHS of 6.8). From (6.5), applying Fubini's theorem, we have

$$
\begin{aligned}
\int_{0}^{\infty} e^{-\lambda u} \nu([u,+\infty[) d u & =\int_{0}^{\infty} e^{-\lambda u} \int_{u}^{\infty} n(v) d v d u \\
& =\int_{0}^{\infty} e^{-\lambda u}\left(\int_{u}^{\infty} \int_{0}^{\infty} e^{-\xi v} \xi d \sigma(\xi) d v\right) d u \\
& =\int_{0}^{\infty} e^{-\lambda u}\left(\int_{0}^{\infty} e^{-\xi u} d \sigma(\xi)\right) d u=\int_{0}^{\infty} \frac{d \sigma(\xi)}{\lambda+\xi}
\end{aligned}
$$

Recall that $x \mapsto m([0, x])$ is an increasing function and $m([0, x]) \underset{x \rightarrow+\infty}{\sim} x^{1 / \beta-1} \kappa(x)$. Then, using Y. Kasahara [Kas76, Lemma 1, p. 73], we have

$$
m^{-1}([0, x]) \underset{x \rightarrow+\infty}{\sim} x^{1 /(1-\beta)-1} \vartheta(x),
$$

where $\vartheta$ is a slowly varying function. By applying [Kas76, Theorem 2, p. 73], 6.9 is seen to be equivalent to

$$
\int_{0}^{\infty} \frac{d \sigma(\xi)}{\lambda+\xi} \underset{\lambda \rightarrow 0}{\sim}(\beta(1-\beta))^{\beta-1} \frac{\Gamma(2-\beta)}{\Gamma(\beta)} \lambda^{-\beta} \widetilde{\vartheta}\left(\frac{1}{\lambda}\right)
$$

where $\widetilde{\vartheta}$ is a slowing varying function such that $\left(x^{1-\beta} \widetilde{\vartheta}(x)\right)^{-1}=x^{\frac{1}{1-\beta}} \vartheta(x)$ (in the sense of composition of functions). Finally, setting

$$
\eta(t):=(\beta(1-\beta))^{\beta-1} \frac{\Gamma(2-\beta)}{\Gamma(\beta)} \widetilde{\vartheta}(t),
$$

and applying the Tauberian Theorem 6.1, we obtain

$$
\nu\left(\left[u,+\infty[) \underset{u \rightarrow+\infty}{\sim} \frac{\eta(u)}{u^{\beta}}\right.\right.
$$

and

$$
\mathbb{E}_{x}\left[e^{-\alpha L_{t}}\right] \underset{t \rightarrow+\infty}{\sim}\left(x+\frac{1}{\alpha}\right) \frac{\eta(t)}{t^{\beta}} .
$$

Note that, from (6.8), we have also proven that

$$
\mathbb{E}_{x}\left[e^{-\alpha L_{t}}\right] \underset{t \rightarrow+\infty}{\sim} \nu\left(\left[t,+\infty[)\left(x+\frac{1}{\alpha}\right) .\right.\right.
$$


Example 1. In the same way as in Section 5, let us consider $\left(X_{t}:=\left(Y_{t}^{(\nu)}\right)^{-2 \nu}\right.$, $t \geq 0)$ where $Y^{(\nu)}$ is a Bessel process of index $\left.\nu \in\right]-1,0[$ reflected at 0 . The speed measure of $X$ is given by

$$
m([0, x])=-\frac{1}{2 \nu(1+\nu)} x^{-1-1 / \nu},
$$

hence, with the notations of Theorem 6.2 $\beta=-\nu$ and $\kappa(x)=-1 /(2 \nu(1+\nu))$. Some easy computations then give

$$
\vartheta(x)=\kappa^{\frac{\nu}{1+\nu}}(x)=\left(-\frac{1}{2 \nu(1+\nu)}\right)^{\frac{\nu}{1+\nu}} \text { and } \eta(x)=\kappa^{-\nu}(x)=\left(-\frac{1}{2 \nu(1+\nu)}\right)^{-\nu}
$$

and, from 6.2,

$$
\mathbb{E}_{x}\left[e^{-\alpha L_{t}}\right] \underset{t \rightarrow+\infty}{\sim}\left(x+\frac{1}{\alpha}\right) \frac{2^{\nu}}{\Gamma(1-\nu)} t^{\nu} .
$$

Note that if $\nu=-1 / 2$ (the Brownian motion case) we get

$$
\mathbb{E}_{x}\left[e^{-\alpha L_{t}}\right] \underset{t \rightarrow+\infty}{\sim}\left(x+\frac{1}{\alpha}\right) \sqrt{\frac{2}{\pi t}} .
$$

Remark 5. A probability measure $\mu$ on $[0,+\infty$ [ is called subexponential if $\mu(] x,+\infty[)$ $>0$ for every $x$, and

$$
\lim _{x \rightarrow+\infty} \frac{\mu^{* 2}(] x,+\infty[)}{\mu(] x,+\infty[)}=2,
$$

where $\mu^{* 2}$ stands for the convolution of $\mu$ with itself. (See Sato Sat99, Chapter 5, p. 164] for other equivalent conditions when $\mu$ is the Lévy measure of a subordinator.) Thus, if we assume that the law of $\frac{1}{\nu(] 1,+\infty[)} \nu_{\mid] 1,+\infty[}$ is subexponential (which is in particular the case if $\nu(] t,+\infty[) \underset{t \rightarrow+\infty}{\sim} t^{-\beta} \eta(t)$ ), this implies (see P. Salminen and P. Vallois [SV09]) that

$$
\mathbb{P}_{x}\left(L_{t}<l\right) \underset{t \rightarrow+\infty}{\sim}(x+l) \nu(] t,+\infty[) .
$$

Therefore, we have directly

$$
\begin{aligned}
\mathbb{E}_{x}\left[e^{-\alpha L_{t}}\right] & =\int_{0}^{1} \mathbb{P}_{x}\left(e^{-\alpha L_{t}}>u\right) d u \\
& =\int_{0}^{\infty} \mathbb{P}_{x}\left(e^{-\alpha L_{t}}>e^{-\alpha l}\right) \alpha e^{-\alpha l} d l \\
& =\int_{0}^{\infty} \mathbb{P}_{x}\left(L_{t}<l\right) \alpha e^{-\alpha l} d l \\
& \sim \sim(] t,+\infty[) \int_{0}^{\infty}(x+l) \alpha e^{-\alpha l} d l \underset{t \rightarrow+\infty}{\sim} \nu(] t,+\infty[)\left(x+\frac{1}{\alpha}\right) .
\end{aligned}
$$


§6.2. Second case: $b<+\infty$ and $m([0, b[)=+\infty$

The resolvent kernel takes the form

$$
R_{\lambda}(0,0)=\int_{0}^{b} \frac{d x}{\Phi^{2}(x, \lambda)} \underset{\lambda \rightarrow 0}{\longrightarrow} b \quad(\text { from } 2.7),
$$

which shows in particular that $X$ is transient. Moreover,

$$
\frac{R_{\lambda}(0, x)}{R_{\lambda}(0,0)}=\Phi(x, \lambda)-\frac{\Psi(x, \lambda)}{R_{\lambda}(0,0)} \underset{\lambda \rightarrow 0}{\sim} 1-\frac{x}{b},
$$

hence we find the equivalent:

$$
\int_{0}^{\infty} e^{-\lambda t} \mathbb{E}_{x}\left[e^{-\alpha L_{t}}\right] d t \underset{\lambda \rightarrow 0}{\sim} \frac{1}{\lambda}\left(1-\left(1-\frac{x}{b}\right) \frac{\alpha}{\alpha+\frac{1}{b}}\right) .
$$

The Tauberian theorem can be applied, and we finally obtain

$$
\mathbb{E}_{x}\left[e^{-\alpha L_{t}}\right] \underset{t \rightarrow+\infty}{\sim} 1-\left(1-\frac{x}{b}\right) \frac{\alpha}{\alpha+\frac{1}{b}} .
$$

§6.3. Third case: $b+m([0, b[)<+\infty$

In this case, to define the diffusion it is necessary to add a supplementary boundary condition at $b$. To this end, let $k(d x)=\frac{1}{k_{0}} \delta_{b}(d x)$ be the killing measure of $X$ (where $\delta_{b}$ stands for the Dirac measure at $b$ ). If $k_{0}=+\infty$, then $X$ is reflected at $b$; this was the subject of Sections 2 to 5 . Therefore, we assume here that $X$ is elastically killed at $b$, i.e. $k_{0}<+\infty$. (Note that $k_{0}=0$ means that $b$ is a killing boundary, i.e. the diffusion, if it hits $b$, is immediately sent to a cemetary state $\partial$; see [BS02, p. 16]). In this set-up, to define the resolvent kernel $R_{\lambda}$, we must start by extending $\Phi$ linearly on $[b,+\infty[$ by setting

$$
\Phi(x, \lambda):=\Phi(b, \lambda)+\Phi^{\prime}(b, \lambda)(x-b) \quad \text { for } x \geq b .
$$

Then the resolvent kernel takes the form

$$
R_{\lambda}(0,0)=\int_{0}^{b+k_{0}} \frac{d x}{\Phi^{2}(x, \lambda)} \underset{\lambda \rightarrow 0}{\longrightarrow} b+k_{0} .
$$

This case is thus very similar to the second one, and the diffusion is again transient. Moreover,

$$
\frac{R_{\lambda}(0, x)}{R_{\lambda}(0,0)} \underset{\lambda \rightarrow 0}{\sim} 1-\frac{x}{b+k_{0}} .
$$

Consequently,

$$
\mathbb{E}_{x}\left[e^{-\alpha L_{t}}\right] \underset{t \rightarrow+\infty}{\sim} 1-\left(1-\frac{x}{b+k_{0}}\right) \frac{\alpha}{\alpha+\frac{1}{b+k_{0}}} .
$$


It is then easy to deduce the law of $L_{\infty}$ :

$$
\mathbb{P}_{x}\left(L_{\infty} \in d u\right)=\frac{x}{b+k_{0}} \delta_{0}(d u)+\left(1-\frac{x}{b+k_{0}}\right) \frac{1}{b+k_{0}} \exp \left(-\frac{u}{k_{0}+b}\right) d u
$$

Example 2. We consider the Brownian motion reflected at 0 and killed at 1 for which $m(d x)=2 d x, b=1$ and $k_{0}=0$. Here, $I=[0,1[$ and we obtain

$$
\mathbb{E}_{x}\left[e^{-\alpha L_{t}}\right] \underset{t \rightarrow+\infty}{\sim} 1-(1-x) \frac{\alpha}{\alpha+1}
$$

and

$$
\mathbb{P}_{x}\left(L_{\infty} \in d u\right)=x \delta_{0}(d u)+(1-x) e^{-u} d u .
$$

Let us remark that, since $L_{\infty}=L_{T_{1}}$ a.s., this entails that under $\mathbb{P}_{0}, L_{T_{1}}$ has an exponential law of parameter 1.

\section{Acknowledgements}

The author would like to thank heartily Professors Pierre Vallois and Bernard Roynette for fruitful discussions.

\section{References}

[BS02] A. N. Borodin and P. Salminen, Handbook of Brownian motion-facts and formulae, 2nd ed., Probab. Appl., Birkhäuser, Basel, 2002. Zbl 1012.60003 MR 1912205

[CPY01] P. Carmona, F. Petit, and M. Yor, Exponential functionals of Lévy processes, in Lévy processes, Birkhäuser Boston, Boston, MA, 2001, 41-55. Zbl 0979.60038 MR 1833691

[Car61] H. Cartan, Théorie élémentaire des fonctions analytiques d'une ou plusieurs variables complexes, Hermann, Paris, 1961. Zbl 0653.30001 MR 0147623

[DM76] H. Dym and H. P. McKean, Gaussian processes, function theory, and the inverse spectral problem, Academic Press, Probab. Math. Statist. 31, New York, 1976. Zbl 0327.60029 MR 0448523

[Fel71] W. Feller, An introduction to probability theory and its applications. Vol. II, 2nd ed., Wiley, New York, 1971. Zbl 0219.60003 MR 0270403

[IM74] K. Itô and H. P. McKean, Diffusion processes and their sample paths, Grundlehren Math. Wiss. 125, Springer, Berlin, 1974. Zbl 0285.60063 MR 0345224

[KK74] I. S. Kac and M. G. Krein, On the spectral functions of the string, Amer. Math. Soc. Transl. 103 (1974), 19-102. Zbl 0291.34017

[Kas76] Y. Kasahara, Spectral theory of generalized second order differential operators and its applications to Markov processes, Japan. J. Math. (N.S.) $1(1975 / 76)$, 67-84. Zbl 0348.60113 MR 0405615

[Kni78] F. B. Knight, On the sojourn times of killed Brownian motion, in Séminaire de Probabilités, XII (Strasbourg, 1976/1977), Lecture Notes in Math. 649, Springer, Berlin, 1978, 428-445. Zbl 0376.60082 MR 0520018

[Kni81] Characterization of the Lévy measures of inverse local times of gap diffusion, in Seminar on Stochastic Processes, 1981 (Evanston, 1981), Progr. Probab. Statist. 1, Birkhäuser Boston, Boston, MA, 1981, 53-78. Zbl 0518.60083 MR 0647781 
[KW82] S. Kotani and S. Watanabe, KreIn's spectral theory of strings and generalized diffusion processes, in Functional analysis in Markov processes (Katata/Kyoto, 1981), Lecture Notes in Math. 923, Springer, Berlin, 1982, 235-259. Zbl 0496.60080 MR 0661628

[Leb72] N. N. Lebedev, Special functions and their applications, Dover Publ., New York, 1972. Zbl 0271.33001 MR 0350075

[Mey66] P.-A. Meyer, Probabilités et potentiel, Actual. Sci. Indust. 1318, Hermann, Paris, 1966. Zbl 0138.10402 MR 0205287

[PY81] J. Pitman and M. Yor, Bessel processes and infinitely divisible laws, in Stochastic integrals (Durham, 1980), Lecture Notes in Math. 851, Springer, Berlin, 1981, 285370. Zbl 0469.60076 MR 0620995

[RY99] D. Revuz and M. Yor. Continuous martingales and Brownian motion, 3rd ed., Grundlehren Math. Wiss. 293, Springer, Berlin, 1999. Zbl 0804.60001|| MR 1303781

[RW00] L. C. G. Rogers and D. Williams, Diffusions, Markov processes, and martingales. Vol. 2, Cambridge Univ. Press, Cambridge, 2000. Zbl 0977.60005 MR 1780932

[RVY06] B. Roynette, P. Vallois, and M. Yor, Some penalisations of the Wiener measure, Japan. J. Math. 1 (2006), 263-290. Zbl 1160.60315 MR 2261065

[SV09] P. Salminen and P. Vallois, On subexponentiality of the Lévy measure of the diffusion inverse local time; with applications to penalizations, Electron. J. Probab. 14 (2009), 1963-1991. Zbl pre05636638 MR 2540855

[Sat99] K. Sato, Lévy processes and infinitely divisible distributions, Cambridge Stud. Adv. Math. 68, Cambridge Univ. Press, Cambridge, 1999. Zbl 0973.60001|| 